Dissipative connections with $U$-shaped steel plate for braces of concentrically braced frames

Peer-reviewed author version

GOUVEIA HENRIQUES, Jose; Calado, L.; Castiglioni, C. A. \& DEGEE, Herve (2019) Dissipative connections with U-shaped steel plate for braces of concentrically braced frames. In: Bulletin of Earthquake Engineering, p. 6203-6237..

DOI: $10.1007 / \mathrm{s} 10518-019-00689-\mathrm{y}$

Handle: http://hdl.handle.net/1942/29821 


\title{
Dissipative connections with U-shaped steel plate for braces of concentrically braced frames
}

\author{
J. Henriques ${ }^{a}$, L. Calado ${ }^{b}$, C. A. Castiglionic ${ }^{c}$ H. Degée ${ }^{a}$ \\ ${ }^{a}$ CERG,IIW, Hasselt Universiteit \\ Campus Diepenbeek, Agoralaan building D, BE3590 Diepenbeek, Belgium \\ ${ }^{b}$ Instituto Superior Técnico, Universidade de Lisboa \\ Campus Alameda, Av. Rovisco Pais,1049-001 Lisboa, Portugal \\ ${ }^{c}$ Dept. of Architecture, Built environment and Construction engineering, Politecnico di \\ Milano \\ Piazza Leonardo da Vinci 32, 20133 Milano, Italy \\ *jose.gouveiahenriques@uhasselt.be, ORCID_0000-0002-8685-233X
}

\begin{abstract}
In concentrically braced frames, braces-to-adjacent member connections are suitable locations for the dissipation of seismic energy. If the implementation of dissipative connections does not compromise the global lateral stiffness of the structure, the compression braces can then be protected from buckling, while the structural ductility is increased and the action effects reduced. Furthermore, an efficient use of dissipative connections allows reducing the cost of post-earthquake interventions. In this paper, an innovative dissipative connection for braced frames is proposed, consisting of a steel plate bent to a $U$ shape and connecting the brace to the adjacent column. In this connection, energy dissipation is obtained through the inelastic flexural deformation that takes place in the plate. This paper presents experimental results on the isolated U-connection and on single-storey concentrically braced frame (real scale) including the U-connection. Besides considering several variations on the geometry of the U-shape plate, the tests considered both monotonic and cyclic loading. The results highlight the efficiency of the U-connection to dissipate the energy input through inelastic deformations. On the other hand, the cyclic tests show however that the connection is potentially sensitive to fatigue, as the deformation capacity is significantly reduced with repeated loading and increasing stress amplitude, requiring thus specific attention in practical design situations.
\end{abstract}

Keywords: U-connection, Dissipative Connection, Concentrically Braced Frame, Deformation Capacity, Fatigue

\section{Introduction}

The main concern in conventional seismic design is the protection of human lives during the earthquake, therefore avoiding structural collapse while allowing significant damage (Priestley M J N, 2000). However, in the last decades the knowledge on the performance of earthquake-resisiting structures has significantly increased leading to a new design philosophy, namely the limitation of structural damage (CEN, 2005b). To this end, the structure is conceived in such a way that the structural response is governed by the so- 
called dissipative components (Dimakogianni D et al., 2015). Damage is then controlled and limited to these parts of the structure, preventing not only the global structural collapse, but ensuring as well the structural integrity and operability. Consequently, costs in post earthquake interventions or due to interruption of operation may be significantly reduced (Morelli F et al., 2017).

When a structure is excited by a seismic action, its effects can be limited through the dissipation of the energy input. There exist different ways to dissipate the seismic energy, such as base isolation, damping or plastic deformations. In most common structures, the dissipation of seismic energy is achieved through plastic deformations. The ductility presented by the governing structural elements, members and connections, reflects then the energy dissipation capacity of the structure. In what concerns ductility, usual structural systems are heterogeoneous, as the different parts of the structure present different level of ductility, ranging from brittle to ductile. It is not economic to design all structural elements as ductile (Landolfo R et al., 2017). Thus, a ductile behavior can only be obtained if the structural response is governed by ductile components. This fundamental requirement can be guaranteed if the capacity design concept is used when designing the structure. This method is nowadays integrated in the seismic design codes (CEN, 2005b) and consists in selecting the ductile elements to govern the structural response and to protect all the others from failure. In order to gurantee failure in the dissipative elements, the actual material properties and the structural overstrength are taken into account (Landolfo R et al., 2017; Fardis M et al., 2005).

Concentrically braced frames (CBF) are commonly used in Europe as seismic resistant steel/composite structures. The structural response consists in a truss behavior where the diagonal bracings are subjected to axial forces. Depending on the structural configuration $(\mathrm{X}, \mathrm{V}$ or inverted $\mathrm{V})$, the dissipation of energy can occur in one or more of the following components: the tensile bracing, the compressive bracing and/or the bracing connections. Although having proved to be a reliable resisting system, the experience shows that strong seismic events can result in significant permament deformations in the braces designed as dissipative members (Bertero V et al., 1994) (Hwang S-H and Lignos D G, 2017) (Tan K G and Christopoulos C, 2016). Subsequently, structural repair involves strengthening or replacement of the damaged braces and requires considerable skill, material and labour cost. A suitable solution to overcome this problem consists in the protection of the braces through the use of anti-seismic systems, such as dissipative connections. In CBF with dissipative connections, the frame incorporates connections between braces and adjacent members providing ductility to the structure, increasing the dissipation of seismic energy and protecting the braces from buckling and yielding. Moreover, as damage is concentrated in the connections, the repair after a strong seismic event becomes easier and less costly. Within the research projects INERD (INERD, 2004) and INNOSEIS (Vayas I et al, 2017), a dissipative connection based on these principles and consisting of a plate bent to a $\mathrm{U}$ shape was developed for application in $\mathrm{CBF}$, connecting the braces to the adjacent columns (Figure 1). The proposed connection is a variant of the U-shape flexural plate (UFP) (Kelly J et al., 1972). It is seen as a suitable solution for low to mid- 
rise buildings providing the possibility of an easy and inexpensive repair after strong earthquakes.

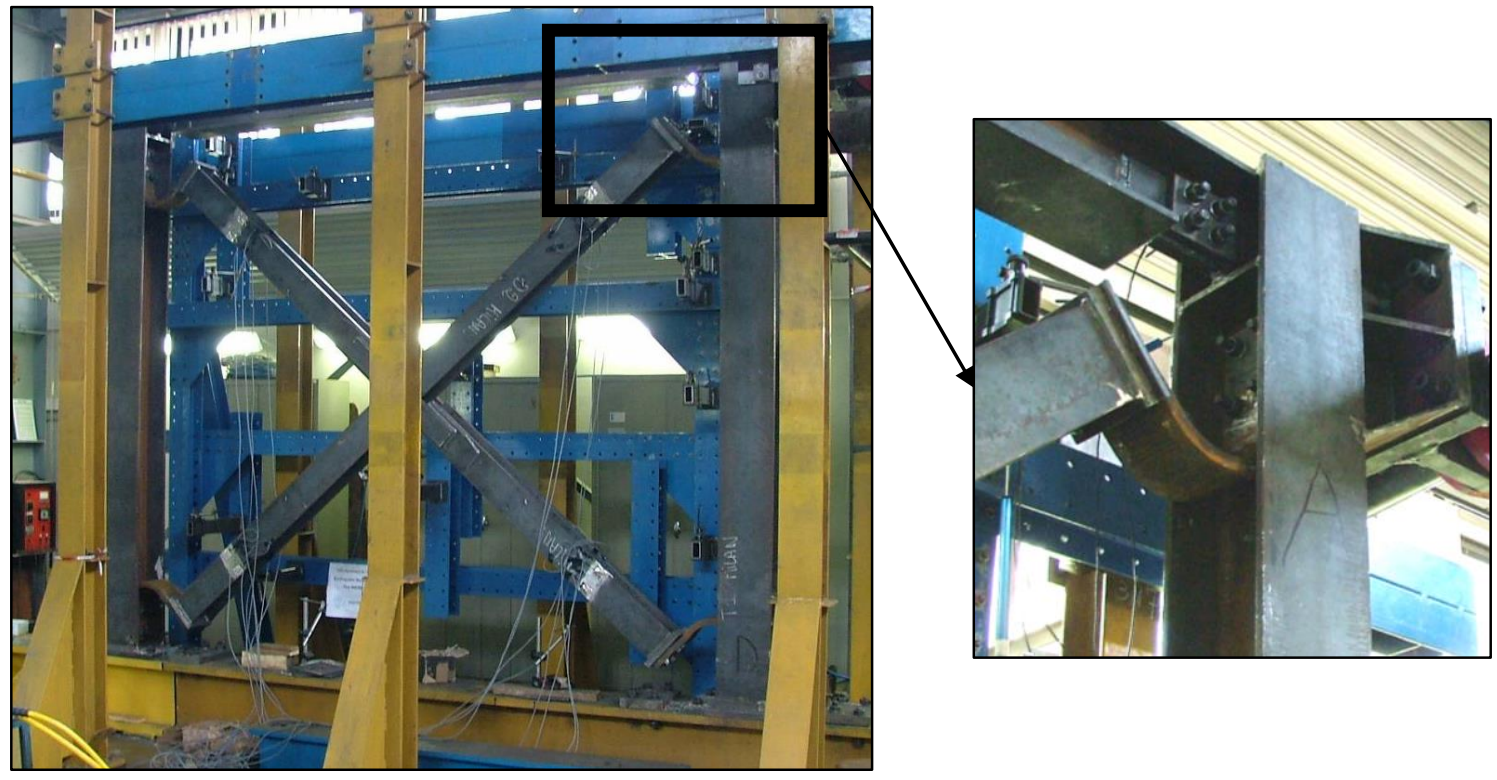

Figure 1: Application of the U-connection on CBF (INERD, 2004)

\section{U-connection for CBF}

The concept of dissipative connections using U-shape flexural plate (UFP) was first introduced by (Kelly J et al., 1972). This connection was developed to be used in coupled shear walls (Schultz A et al., 1994a; Schultz A et al., 1994b) where the seismic energy is dissipated by the inelastic bending of the $U$-shape plate subjected to shear forces between the coupled walls. This energy dissipation system has been successfully used in different projects considering different structural materials such as precast concrete and timber (Priestley M J N et al., 1999; Palermo A et al., 2005; Pampanin S et al., 2011). Besides the significant energy dissipation capacity, the UFP has the advantage of being easy to fabricate, therefore being low cost, easy to replace (rehabilition) and exhibititing a stable hysteretic behavior (Baird A et al., 2014). An efficient application of the UFP has been proposed and implemented in High-Rise buildings by Nüyü_tek (Nüyün_tek, 2018). The system (Figure 2-b) consists in $\mathrm{X}$ bracing in which the diagonal braces, covering several floors, are connected at the intersection point by a horizontal dissipative system comprising two plates, where the braces are fixed and connected together by UFPs welded to these plates. The seismic forces resisted by the bracing system induce a shear force in the referred dissipative system leading to the dissipation of energy through the flexural response of the UFPs, as proposed in (Kelly J et al., 1972). This dissipation system provides a high damping effect to the buildings, reducing significantly the lateral movements and consequently the interstorey drifts. The high efficiency of this seismic protection system was demonstrated during the strong Earthquake in Chile 2010. The dissipative system that was implemented in the construction of the tallest building built in Santiago (Chile), i.e. the "Titanium - La Portada" (Barroilhet A, 2011), provided an 
efficient seismic protection, not only avoiding collapse but also limiting the structural and non-structural damages guaranteeing the immediate operation of the building after the earthquake.

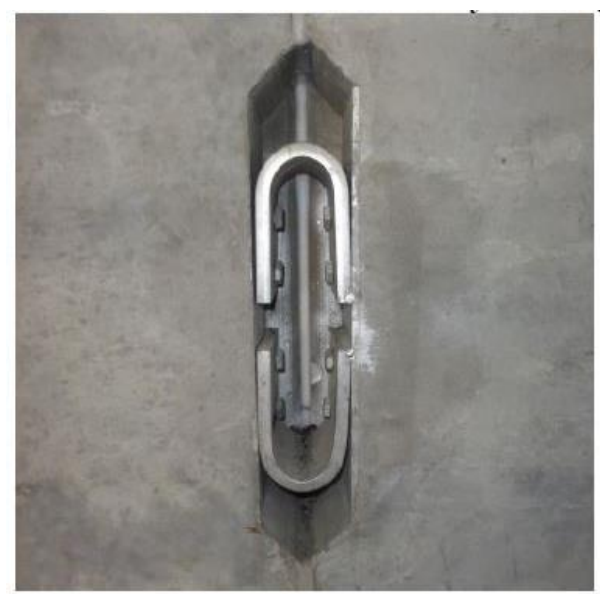

a) Dissipative joint connection of coupled precast shear walls using UFP (Baird A et al., 2014)

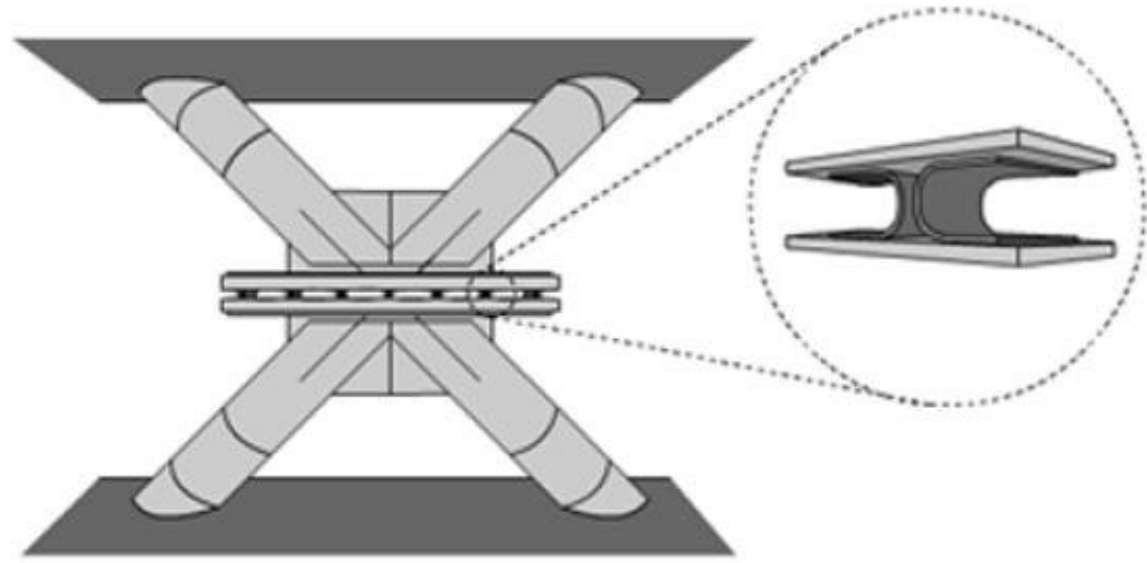

b) Dissipative bracing system implementing the UFP dissipative connection (Jensen $\mathrm{H}$ et al., 2012)

Figure 2: U-shape Flexural Plate (UFP) applications

The U-connection proposed within the research project INERD (INERD, 2004) uses the same basic principle of energy dissipation as in UFP: dissipation of energy through the inelastic flexural deformation of the U-shaped plate. Though, a different application is foreseen. In the UFP the bent part realizes an half circle requiring that the "sliding" plates of the connected members are disposed in a parallel position. In the U-connection, the Ushaped plate is fabricated with a varying angular position between the connecting faces (hereon denominated as "legs"), reflecting the different orientation of the connected members. This leads to the fact that the loading conditions differ quite significantly from the original UFP. Hence, it is the purpose of this paper to present the experimental programme executed on this specific dissipative U-connection. Tests were performed on isolated U-connections (U-shaped plates) and on real scale CBFs incorporating the Uconnection. The mechanical response of the U-connection subjected to both monotonic and cyclic loading is here presented. Under monotic loading, the test results demonstrate 
the high energy dissipation capacity of the connection. While under cyclic loading, the connection exposes its sensitivity to fatigue, limiting its deformation capacity.

The U-connection consists of a thick steel plate bent to a U-shape, from here on denominated U-plate, connected to the bracings and adjacent members by means of bolts. As for the UFP, energy is dissipated through the inelastic bending deformation within the U-plate. The U-plate is simple to fabricate and nevertheless an efficient solution to control the inelastic deformations of CBFs subjected to seismic motion. Through the appropriate design of the U-plate, inelastic deformations can be controlled and limited within the Uplate while the other parts of the U-connection, the bracing and the adjacent members remain in the elastic regime.

The application of the U-connection to a CBF is illustrated in Figure 3. Two relative positions of the U-plate are possible resulting in two distinct loading conditions: i) parallel loading (Figure 3-a); ii) transverse loading (Figure 3-b). When the load is applied in the plane of the U-plate "leg", the load is denominated as parallel, and when transverse to the plane of the U-plate "leg", the load is denominated as transverse. These denominations will be used hereon. The position of the U-plate does not only affect the type of loading but also the type of connection between the U-plate and the bracing. Accordingly, for parallel loading configuration, an overlap connection (Figure 3-a) can be used between the U-plate and the brace, while for transverse loading configuration, an end-plate connection (Figure 3-b) should be used.

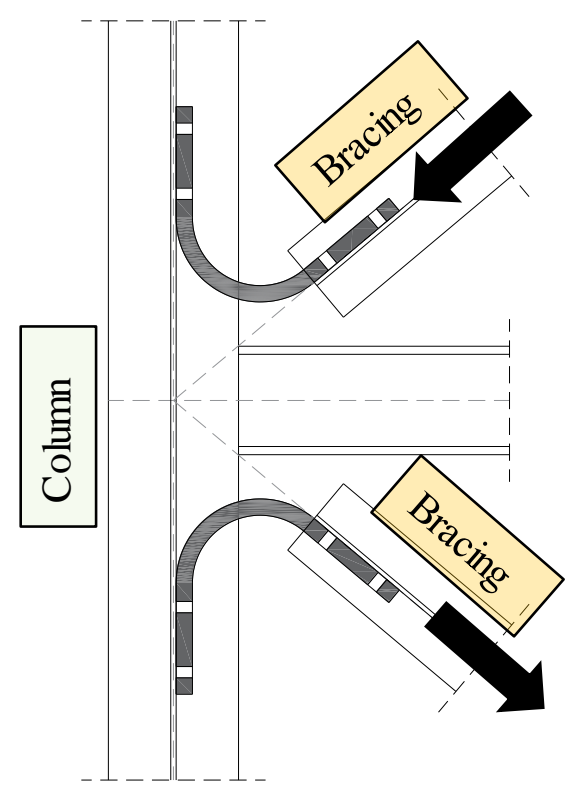

a) Single overlap connection, U-brace parallel

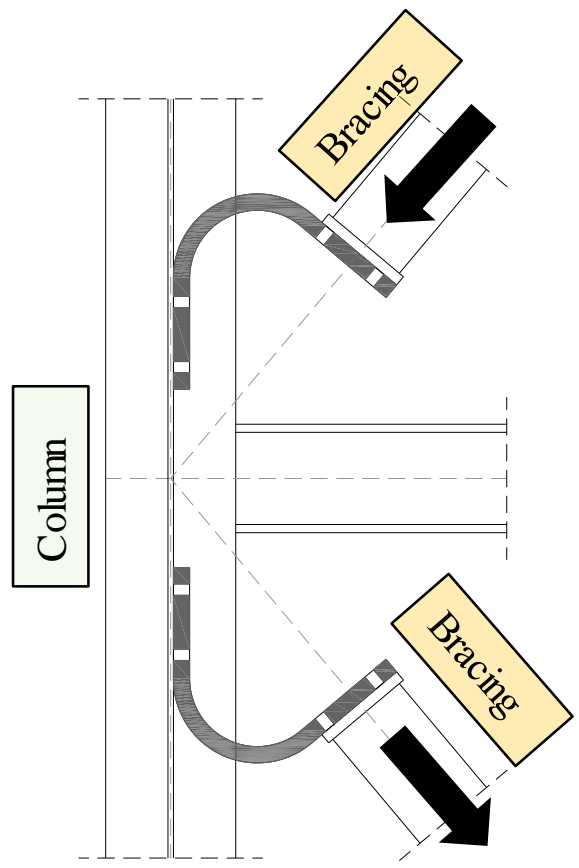

b) End-plate connection, U-brace transverse Figure 3: U-connection concept

The U-connection can be easily adapted to any frame geometry. The angle between the connected members can easily be matched in the fabrication of the U-plate by varying the angle between the connecting "legs" accordingly. Furthermore, the mechanical properties can be controlled adjusting the following geometric properties: bending radius; thickness 
of the U-plate; angle between brace and adjacent member; width of the U-plate; length of the straight parts; and number of bolts to perform the connection between the U-plate and the adjacent members.

\section{Experimental tests on U-connections}

\subsection{General}

The experimental tests on isolated U-connection were performed at IST in Lisbon, Portugal (INERD, 2004). To characterize the mechanical behavior, according to the loading type, two group of tests were executed: one group under monotonic loading and another with cyclic loading. Coupon tests were also executed to obtain the real mechanical properties of the steel used in the fabrication of the U-plate. Based on the results of the cyclic loading tests, fatigue design provisions, in accordance with the EN 1993-1-9 (CEN, 2005a), were proposed. In the following sections, the referred experimental testing programme and the obtained results are summarized.

\subsection{Test specimens, Test programme and Monitoring}

In order to characterize the U-connection as dissipative component, the conducted tests focused on the testing of the U-plate and therefore the other parts of the U-connection, as the bolts, the end-plate (for the transverse loading) and the connected members, were overdesigned to remain in the elastic domain. In Figure 4 all the geometric properties required to fabricate the U-plate are identified. The represented parameters have the following meaning:

- $R$ - bend radius;

- $\alpha$ - angle between U-plate "legs",

- $e$-thickness of the U-plate;

- $h$ - length of straight faces (U-plate "legs");

- $\quad b$ - width of the U-plate;

- $\quad e_{1}-$ end distance of the bolts;

- $e_{2}$ - edge distance of the bolts;

- $p_{1}$ - bolts spacing in the load direction;

- $\quad p_{2}-$ bolt spacing in the perpendicular direction to the loading.

In Table 1 and Table 2, the main geometric and material properties of the test specimens are summarized, respectively. 


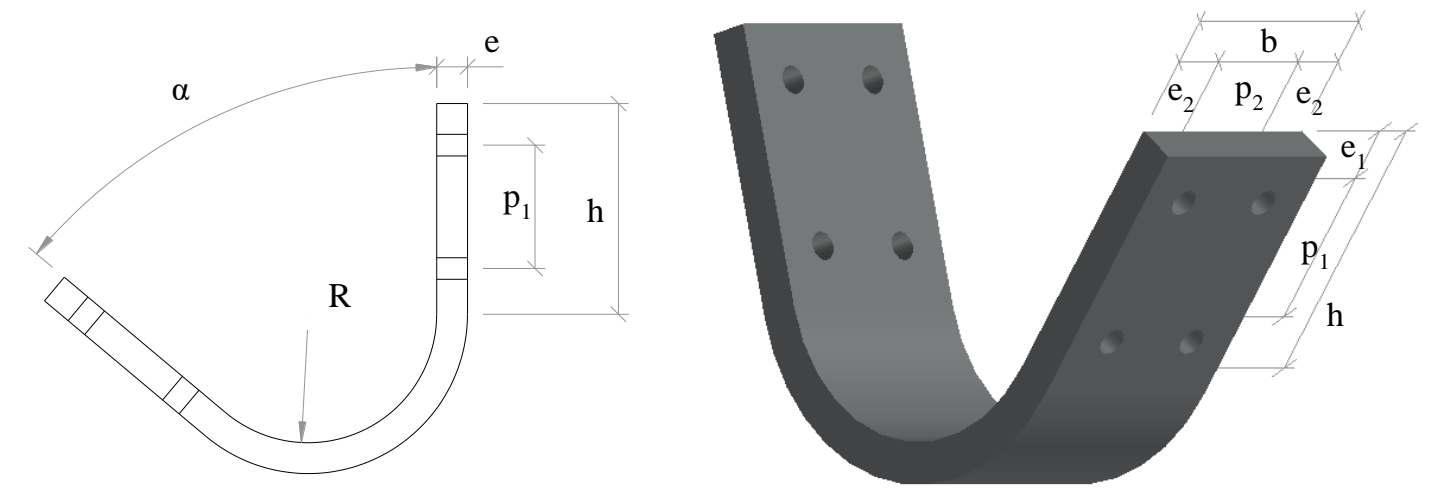

Figure 4: U Plate geometric parameters

Table 1: Summary of the geometric properties of the test specimens (U Plate) and range of variation

\begin{tabular}{|c|c|c|c|c|c|c|c|c|}
\hline $\mathbf{R}[\mathbf{m m}]$ & $\begin{array}{c}\boldsymbol{\alpha} \\
{\left[{ }^{\mathbf{0}}\right]}\end{array}$ & $\begin{array}{c}\mathbf{e} \\
{[\mathbf{m m}]}\end{array}$ & $\begin{array}{c}\mathbf{b} \\
{[\mathbf{m m}]}\end{array}$ & $\begin{array}{c}\mathbf{h} \\
{[\mathbf{m m}]}\end{array}$ & $\begin{array}{c}\mathbf{e}_{1} \\
{[\mathbf{m m}]}\end{array}$ & $\begin{array}{c}\mathbf{e}_{2} \\
{[\mathbf{m m}]}\end{array}$ & $\begin{array}{c}\mathbf{p}_{1} \\
{[\mathbf{m m}]}\end{array}$ & $\begin{array}{c}\mathbf{p}_{2} \\
{[\mathbf{m m}]}\end{array}$ \\
\hline $100 ; 125$ & $30 ; 39 ; 45 ; 50$ & $25 ; 30$ & 160 & 205 & 40.5 & 40 & 120 & 80 \\
\hline
\end{tabular}

Table 2: Mechanical properties of the test specimens' materials

\begin{tabular}{|c|c|}
\hline Steel Plate (U-plate) & Bolts \\
\hline S355 & \\
\hline Coupon Tests: & M20-8.8 \\
Avg $\mathrm{R}_{\mathrm{eL}}=358 \mathrm{~N} / \mathrm{mm}^{2}$ & $\mathrm{M} 24-12.9$ \\
Avg $\mathrm{R}_{\mathrm{eH}}=386 \mathrm{~N} / \mathrm{mm}^{2}$ & \\
Avg $\mathrm{R}_{\mathrm{m}}=621 \mathrm{~N} / \mathrm{mm}^{2}$ & \\
\hline
\end{tabular}

The testing programme considered both loading configurations, the loading acting parallel and transverse to the U Plate "legs", as described in $\S 2$. The test layout is illustrated in Figure 5 for the case of parallel loading. The load was applied to the test specimens by means of a semi-automatic actuator and using a displacement control. For each test specimen, different loading protocols were considered and consisted in: i) monotonic load applied in order to further bend the U-plate (hereon denominated compression); ii) monotonic load applied in order to "unfold" the U-plate (hereon denominated tension); iii) cyclic loading following the ECCS cyclic loading protocol (ECCS, 1986); iv) to vii) cyclic loading with constant amplitude of imposed deformation, respectively, $20 \mathrm{~mm}, 40 \mathrm{~mm}, 60 \mathrm{~mm}$ and $80 \mathrm{~mm}$. The results of both monotonic tests were used to define the cyclic loading protocol given in (ECCS, 1986). In Table 3 is summarized the complete experimental programme on the isolated U-plate. 


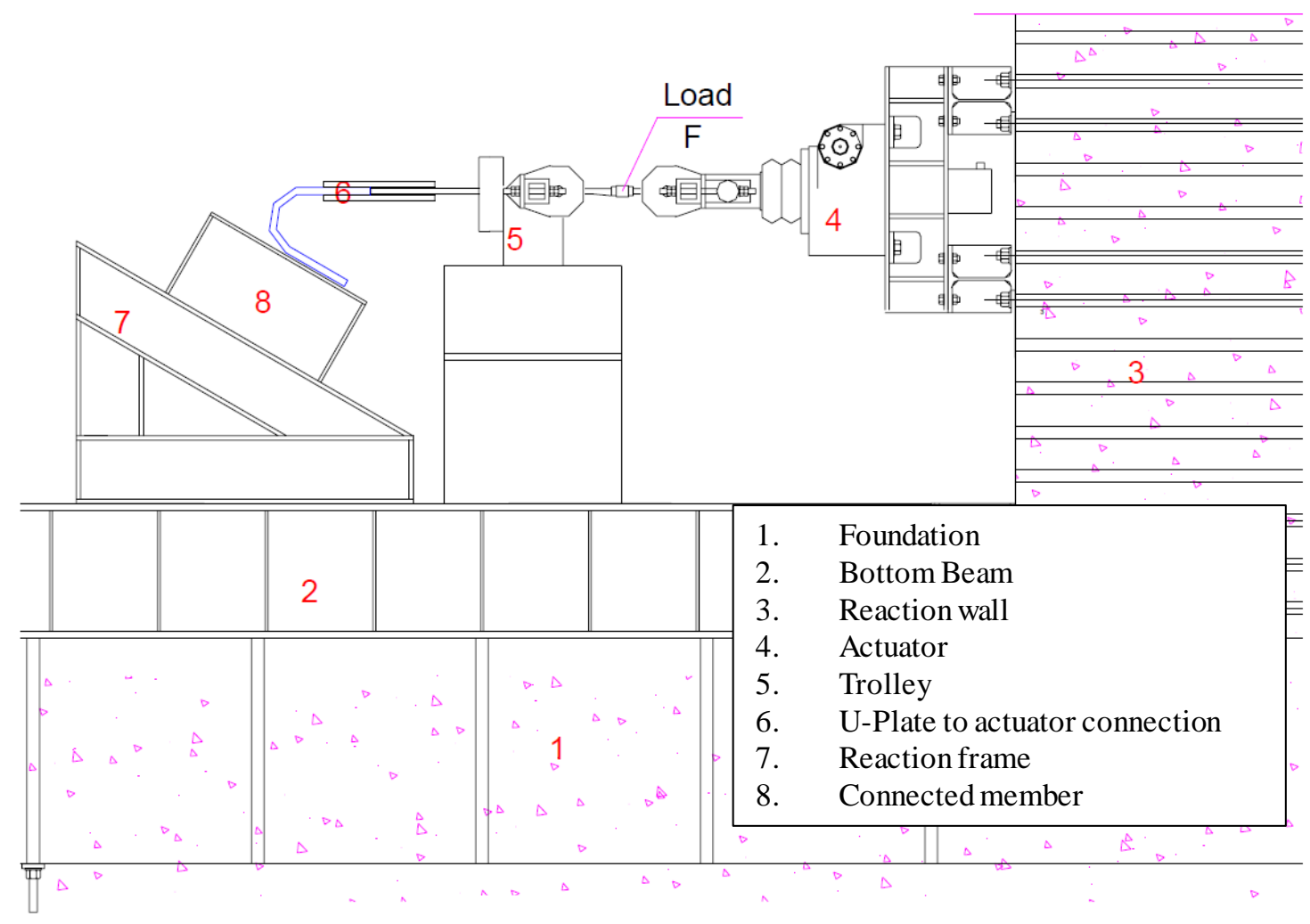

Figure 5: Scheme of the test layout (for parallel loading)

Table 3: Test programme on isolated U Plate

\begin{tabular}{|c|c|c|c|c|c|}
\hline $\begin{array}{c}\text { Test } \\
\text { Specimen ID }\end{array}$ & $\mathbf{R}[\mathbf{m m}]$ & e $[\mathrm{mm}]$ & $\alpha\left[^{0}\right]$ & Position & $N^{0}$ of tests \\
\hline Test 1 & 100 & 25 & 45 & \multirow{7}{*}{$\begin{array}{l}\text { Parallel } \\
\text { Loading }\end{array}$} & \multirow{11}{*}{$\begin{array}{c}\text { For all specimes } \\
2 \text { monotonic } \\
\text { loading and } 4 \\
\text { cyclic loading }\end{array}$} \\
\hline Test 2 & 100 & 25 & 50 & & \\
\hline Test 3 & 100 & 30 & 50 & & \\
\hline Test 4 & 125 & 30 & 50 & & \\
\hline Test 5 & 125 & 25 & 30 & & \\
\hline Test 6 & 125 & 25 & 45 & & \\
\hline Test 7 & 125 & 25 & 50 & & \\
\hline Test 8 & 125 & 25 & 30 & \multirow{4}{*}{ 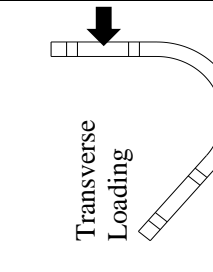 } & \\
\hline Test 9 & 125 & 25 & 39 & & \\
\hline Test 10 & 125 & 25 & 45 & & \\
\hline Test 11 & 125 & 30 & 39 & & \\
\hline
\end{tabular}

The test data acquisition system consisted in one LVDT to evaluate the displacement in the direction of the actuator (in reality corresponding to the axial direction of the bracing) and the load cell of the actuator. 


\subsection{Summary of the test results}

\subsubsection{Monotonic loading}

For each test, a force-displacement curve was obtained which reflects the response of the U-plate under both loading conditions (parallel and transverse) and in both directions (tension and compression). In Figure 6 are shown and compared the force-displacement curves for two configurations (Test 6 and Test 10, see Table 3), where the testing variable is the position of the loading, parallel or transverse. The force represents the force that was applied by the actuator. Thus, it reproduces the axial force on the bracing. The positive value represents compression and the negative tension, as explained in §3.2. Then, the displacement is the deformation in the direction of the load application. From this comparison, two first conclusions can be drawn: i) the U-plate, presents a higher load and deformation capacity when loaded in compression and ii) the load capacity is higher for the U-plate with transverse loading. In Figure 7, the deformed shapes of the U-plates after the different monotonic tests are shown. There is a similarity in both types of loading position: i) when the loading is in compression (Figure 7 a) and c)), the failure occurs in the gross-cross section due to extensive bending of the plate; ii) when the loading is in tension (Figure $7 \mathrm{~b}$ ) and d)), failure occurs in the net-cross section (bolted zone), due to the high tension stresses that develops in this region. This is due to the different deformation patterns, according to loading position on the U-plate and explains the lower deformation capacity observed in the tension loading tests. The net-section failure is indeed less ductile than a failure occurring in the gross cross-section.

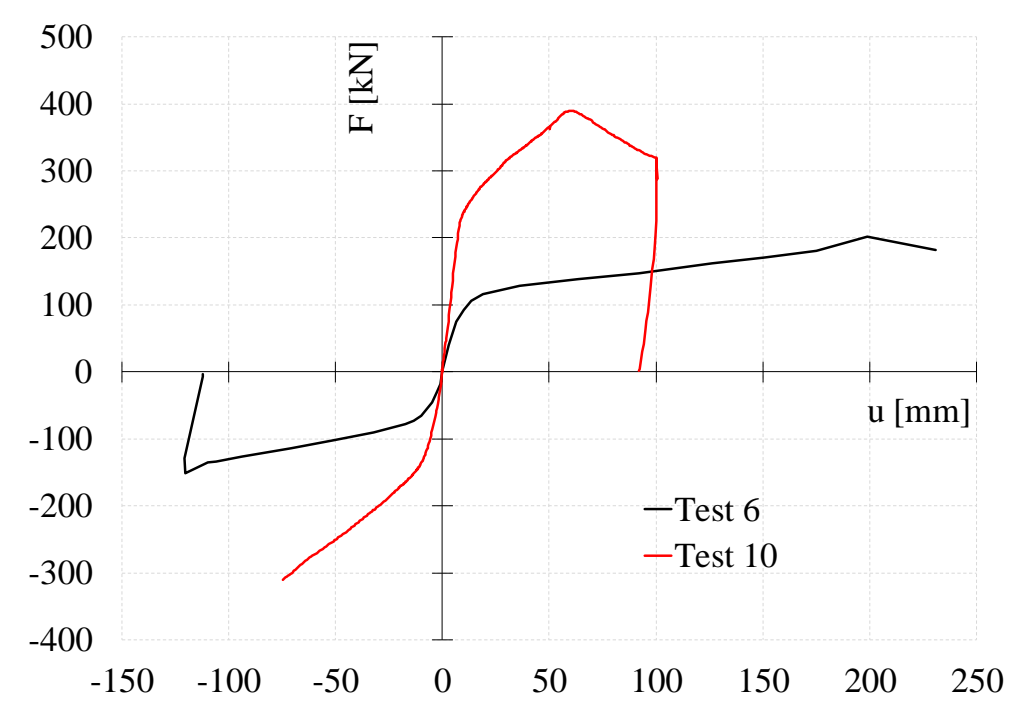

Figure 6: Force-displacement curve obtained in the tests with Monotonic loading 


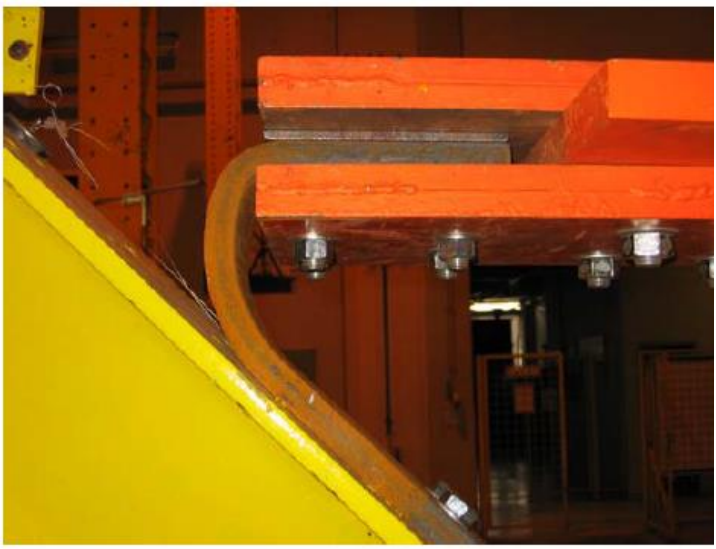

a) Test 6 - Compression loading

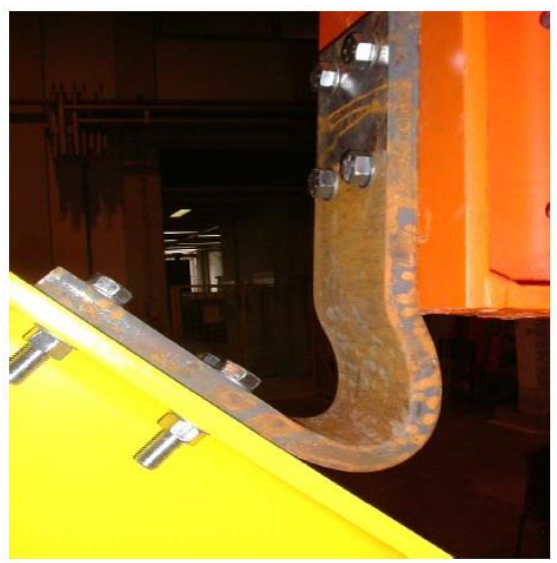

c) Test 10 - Compression loading

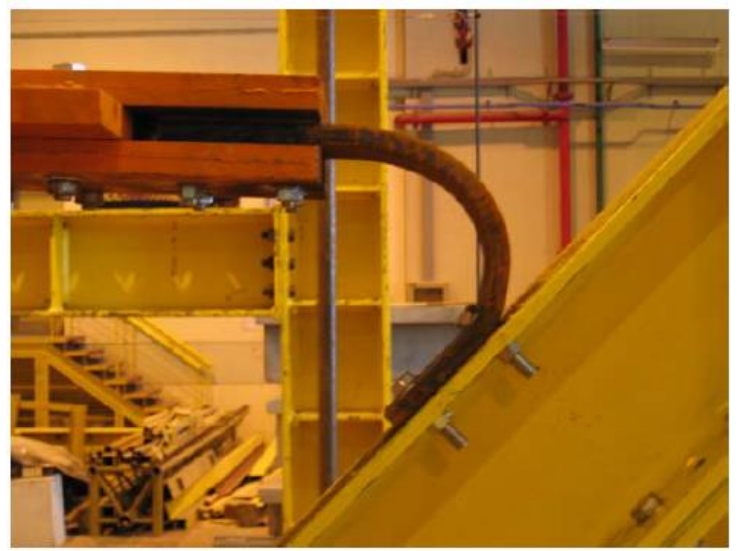

b) Test 6 - Tension loading

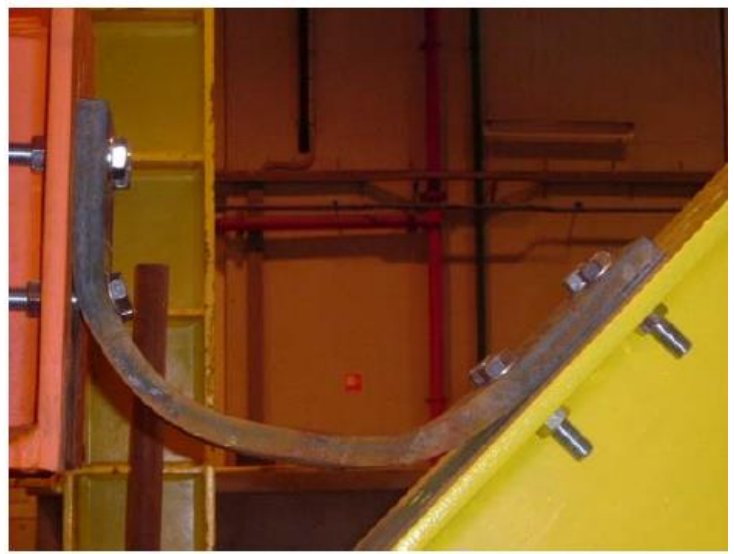

d) Test 10 - Tension loading

Figure 7: Failure of connections in the monotonic tests

A total of 22 monotonic tests were performed. For each configuration, one compression and one tension loading test was executed. Only for test specimen Test 4 subjected to tension loading data was not acquired due to some problems during the test. Given the amount of data produced, in Table 4 is given a summary of the main mechanical properties to characterize the behavior of the U-plate subjected to monotonic loading. The presented properties have the following meaning:

- $u_{y}$ - elastic limit deformation;

- $F_{y}$ - elastic limit force;

- $S$ - initial stiffness;

- $u_{\max }$ - maximum deformation;

- $F_{\max }$ - maximum force;

- Plastic Energy - absorbed energy resulting from inelastic deformations;

- Total Energy - absorbed energy resulting from elastic and inelastic deformations. The deformations $\left(u_{y}\right.$ and $u_{\max }$ ) consist in the deformation of the U-plate in the direction of loading which in a structure should correspond to the axial deformation of the brace. The load is the one applied by the actuator, therefore it represents the load capacity of the U-plate (that can be transferred by the brace). Note that the parameter $u_{\max }$ represents the maximum deformation measured in the test and does not correspond to the deformation 
at maximum force $\left(F_{\max }\right)$. A detailed comparison between the different mechanical parameters and the testing variables is given later in $\S 5.1$.

Table 4: Summary of the monotonic tests

\begin{tabular}{|c|c|c|c|c|c|c|c|c|}
\hline Test ID & $\begin{array}{l}\text { Type of } \\
\text { loading }\end{array}$ & $\mathbf{u}_{\mathbf{y}}[\mathbf{m m}]$ & $\mathbf{F}_{\mathbf{y}}[\mathrm{kN}]$ & $\begin{array}{c}\mathrm{S} \\
{[\mathrm{kN} / \mathrm{m}]}\end{array}$ & $\begin{array}{c}\mathbf{u}_{\max } \\
{[\mathrm{mm}]}\end{array}$ & $\begin{array}{l}\mathbf{F}_{\max } \\
{[\mathbf{k N}]}\end{array}$ & $\begin{array}{c}\text { Plastic } \\
\text { Energy } \\
{[\mathrm{kNm}]}\end{array}$ & $\begin{array}{c}\text { Total } \\
\text { Energy } \\
{[\mathrm{kNm}]}\end{array}$ \\
\hline \multirow{2}{*}{ Test 1} & Compr. & 12.4 & 133.0 & 10716.2 & 175.6 & 257.0 & 22.3 & 24.7 \\
\hline & Tension & 10.6 & 98.0 & 9230.1 & 174.5 & 258.0 & 14.6 & 17.2 \\
\hline \multirow{2}{*}{ Test 2} & Compr. & 10.2 & 144.0 & 14158.0 & 150.2 & 258.0 & 16.2 & 18.0 \\
\hline & Tension & 7.8 & 90.0 & 11493.6 & 150.9 & 240.0 & 11.3 & 12.8 \\
\hline \multirow{2}{*}{ Test 3} & Compr. & 11.3 & 217.0 & 19096.0 & 153.4 & 375.0 & 24.3 & 27.5 \\
\hline & Tension & 11.7 & 153.0 & 13106.5 & 134.7 & 293.0 & 18.6 & 21.7 \\
\hline \multirow{2}{*}{ Test 4} & Compr. & 10.8 & 172.0 & 15913.2 & 191.5 & 205.0 & 17.0 & 19.5 \\
\hline & Tension & - & - & - & - & - & - & - \\
\hline \multirow{2}{*}{ Test 5} & Compr. & 18.4 & 96.0 & 5198.3 & 170.3 & 139.0 & 8.8 & 10.5 \\
\hline & Tension & 9.9 & 63.0 & 6398.8 & 190.3 & 153.0 & 2.9 & 4.5 \\
\hline \multirow{2}{*}{ Test 6} & Compr. & 11.6 & 111.0 & 9598.9 & 231.2 & 202.0 & 14.6 & 16.4 \\
\hline & Tension & 10.0 & 75.0 & 7555.3 & 120.7 & 151.0 & 11.1 & 12.3 \\
\hline \multirow{2}{*}{ Test 7} & Compr. & 19.0 & 130.0 & 6824.6 & 119.7 & 174.0 & 14.3 & 16.6 \\
\hline & Tension & 13.2 & 77.8 & 5912.0 & 150.2 & 209.0 & 9.5 & 12.4 \\
\hline \multirow{2}{*}{ Test 8} & Compr. & 10.8 & 238.9 & 22051.1 & 120.2 & 412.0 & 18.1 & 21.6 \\
\hline & Tension & 11.0 & 127.8 & 11572.9 & 124.5 & 327.0 & 19.1 & 22.7 \\
\hline \multirow{2}{*}{ Test 9} & Compr. & 12.9 & 260.0 & 20096.6 & 120.1 & 361.0 & 18.3 & 22.3 \\
\hline & Tension & 12.3 & 127.8 & 10346.6 & 100.8 & 334.0 & 16.2 & 21.6 \\
\hline \multirow{2}{*}{ Test 10} & Compr. & 9.4 & 257.8 & 27515.3 & 100.5 & 389.0 & 18.6 & 21.2 \\
\hline & Tension & 10.2 & 146.7 & 14316.2 & 74.7 & 311.0 & 12.3 & 15.7 \\
\hline \multirow{2}{*}{ Test 11} & Compr. & 11.8 & 390.0 & 12008.4 & 114.1 & 540.0 & 23.6 & 29.1 \\
\hline & Tension & 17.1 & 205.6 & 33038.7 & 70.6 & 287.0 & 5.2 & 8.6 \\
\hline
\end{tabular}

\subsubsection{Cyclic loading}

In the cyclic loading tests, for each configuration of the U-plate, 5 different loading strategies were used, resulting in a total of 55 tests. In Figure 8, the force-deformation loops for test specimens Test 6 and Test 10 obtained from tests using the ECCS cyclic loading protocol (ECCS, 1986) are presented. The difference between these two specimens is the loading position to the U-plate, parallel and transverse, respectively. The curves confirm the lower load capacity of the U-plate when loaded in tension. On the other hand, the hysteretic loops highlight a progressive degradation of the properties when the U-plate is loaded in compression (pinching phenomenon). Thus, a loss of dissipation of energy is observed. Comparing the deformation capacity observed in both type of tests, monotonic and cyclic, a reduction is observed indicating that the U-plate is sensitive to fatigue. This observation is more evident for test specimen Test 6 . In what concerns the modes of failure, net-section failure was observed in both tests (Figure 9). As for the monotonic tests, in Table 5 and Table 6 
are summarized the mechanical properties obtained from all cyclic tests. The total number of cycles is also reported. In Table 7 is given the cumulative absorbed energy for each test specimen and for each cyclic loading protocol. The absorbed energy decreases with the cycle amplitudes which is justified by the fatigue failure of the connection. As it can be confirmed by the total number of cycles up to failure given in Table 5 and Table 6 , the higher is the amplitude of the cycles, and consequently of the imposed deformation range, the lower is the number of cycles to failure. A detailed and comparative analysis of these results is presented later in $\$ 5.2$.

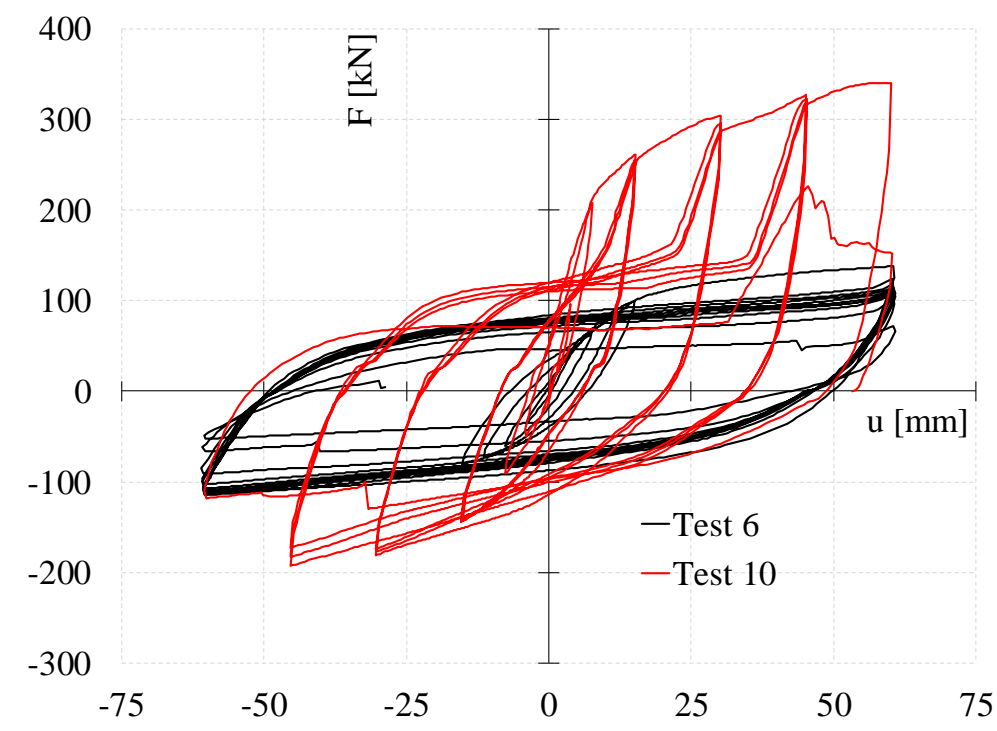

Figure 8: Response of the U-connection under cyclic loading (ECCS cyclic loading protocol)

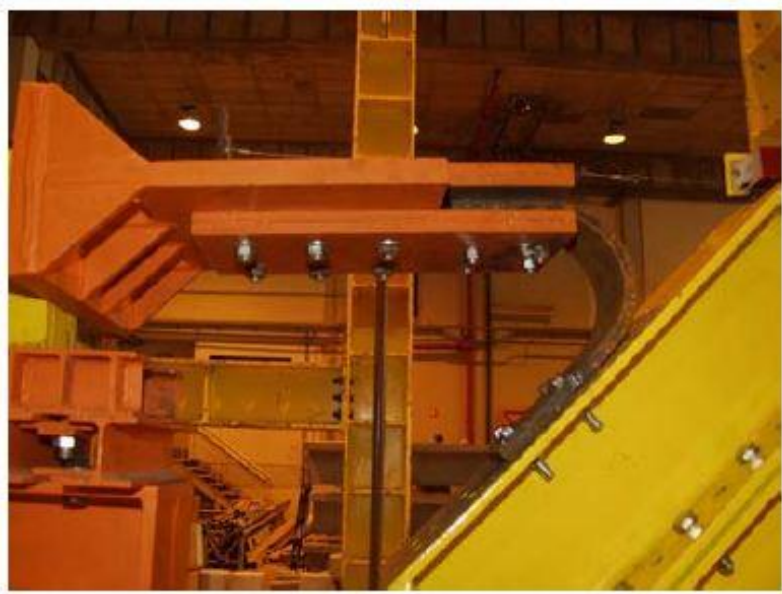

a) Test 6

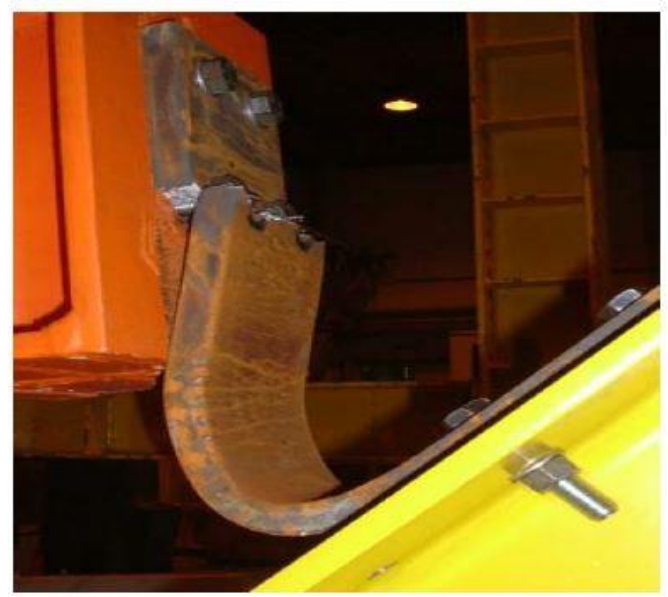

b) Test 10

Figure 9: Failure of connections in the cyclic tests (ECCS cyclic loading protocol) 
Table 5: Summary of the cyclic loading tests

\begin{tabular}{|c|c|c|c|c|c|c|c|c|}
\hline $\begin{array}{l}\text { Test } \\
\text { ID }\end{array}$ & $\begin{array}{l}\text { Loading } \\
\text { Protocol }\end{array}$ & $\begin{array}{c}\text { Fy } \\
{[\mathrm{kN}]}\end{array}$ & $\begin{array}{c}\mathrm{F}_{\max } \\
\text { (Comp.) } \\
{[\mathrm{kN}]}\end{array}$ & $\begin{array}{c}\mathrm{F}_{\max } \\
\text { (Tension) } \\
{[\mathrm{kN}]}\end{array}$ & $\begin{array}{c}\mathrm{u}_{\mathrm{y}} \\
{[\mathrm{mm}]}\end{array}$ & $\begin{array}{c}\mathrm{u}_{\max } \\
(\text { Comp.) } \\
{[\mathrm{mm}]}\end{array}$ & $\begin{array}{c}\mathrm{u}_{\max } \\
\text { (Tension) } \\
{[\mathrm{mm}]}\end{array}$ & $\begin{array}{l}\text { Total Number of } \\
\text { Cycles to Failure }\end{array}$ \\
\hline \multirow{5}{*}{$\overrightarrow{\breve{s}}$} & ECCS & 94.0 & 165.0 & 133.0 & 8.1 & 75.5 & 75.6 & 16 \\
\hline & $\Delta=20 \mathrm{~mm}$ & 81.0 & 139.0 & 108.0 & 6.4 & 20.9 & 20.9 & 81 \\
\hline & $\Delta=40 \mathrm{~mm}$ & 88.0 & 159.0 & 125.0 & 6.6 & 40.9 & 40.9 & 21 \\
\hline & $\Delta=60 \mathrm{~mm}$ & 99.0 & 170.0 & 137.0 & 8.6 & 61.1 & 60.9 & 13 \\
\hline & $\Delta=80 \mathrm{~mm}$ & 90.0 & 180.0 & 144.0 & 7.7 & 80.7 & 84.0 & 9 \\
\hline \multirow{5}{*}{ 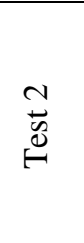 } & ECCS & 94.1 & 165.0 & 133.0 & 8.0 & 75.5 & 75.7 & 16 \\
\hline & $\Delta=20 \mathrm{~mm}$ & 82.0 & 145.0 & 111.0 & 6.6 & 20.9 & 21.1 & 89 \\
\hline & $\Delta=40 \mathrm{~mm}$ & 91.5 & 173.0 & 134.0 & 6.4 & 40.8 & 40.9 & 19 \\
\hline & $\Delta=60 \mathrm{~mm}$ & 95.0 & 180.0 & 144.0 & 7.6 & 60.7 & 60.9 & 12 \\
\hline & $\Delta=80 \mathrm{~mm}$ & 95.0 & 180.0 & 144.0 & 7.6 & 60.7 & 60.9 & 10 \\
\hline \multirow{5}{*}{ 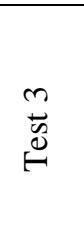 } & ECCS & 162.0 & 240.0 & 205.0 & 11.7 & 75.3 & 75.7 & 17 \\
\hline & $\Delta=20 \mathrm{~mm}$ & 133.0 & 234.0 & 169.0 & 6.7 & 21.2 & 20.9 & 63 \\
\hline & $\Delta=40 \mathrm{~mm}$ & 169.0 & 250.0 & 203.0 & 11.6 & 40.7 & 40.8 & 16 \\
\hline & $\Delta=60 \mathrm{~mm}$ & 142.0 & 271.0 & 204.0 & 8.5 & 60.8 & 60.8 & 10 \\
\hline & $\Delta=80 \mathrm{~mm}$ & 171.0 & 285.0 & 200.0 & 11.2 & 80.6 & 80.4 & 7 \\
\hline \multirow{5}{*}{$\stackrel{+}{\stackrel{+}{0}}$} & ECCS & 99.0 & 207.0 & 163.0 & 7.1 & 75.6 & 60.7 & 15 \\
\hline & $\Delta=20 \mathrm{~mm}$ & 98.0 & 181.0 & 130. & 5.9 & 21.0 & 20.9 & 70 \\
\hline & $\Delta=40 \mathrm{~mm}$ & 130.0 & 207.0 & 155.0 & 13.5 & 40.7 & 40.9 & 26 \\
\hline & $\Delta=60 \mathrm{~mm}$ & 114.0 & 206.0 & 166.0 & 9.0 & 60.6 & 60.8 & 14 \\
\hline & $\Delta=80 \mathrm{~mm}$ & 132.0 & 217.0 & 178.0 & 11.9 & 80.5 & 80.9 & 9 \\
\hline \multirow{5}{*}{$\stackrel{n}{n}$} & ECCS & 72.0 & 116.0 & 102.0 & 11.5 & 90.8 & 75.9 & 18 \\
\hline & $\Delta=20 \mathrm{~mm}$ & 58.0 & 86.0 & 70.0 & 8.7 & 21.8 & 21.5 & 191 \\
\hline & $\Delta=40 \mathrm{~mm}$ & 69.0 & 105.0 & 87.0 & 11.5 & 41.0 & 41.1 & 40 \\
\hline & $\Delta=60 \mathrm{~mm}$ & 69.0 & 97.0 & 115.0 & 9.8 & 61.0 & 61.1 & 19 \\
\hline & $\Delta=80 \mathrm{~mm}$ & 77.0 & 121.0 & 103.0 & 12.7 & 80.7 & 81.0 & 13 \\
\hline \multirow{5}{*}{$\begin{array}{l}0 \\
\vec{w} \\
\stackrel{0}{0}\end{array}$} & ECCS & 82.0 & 132.0 & 112.0 & 10.5 & 75.8 & 75.9 & 16 \\
\hline & $\Delta=20 \mathrm{~mm}$ & 75.0 & 114.0 & 86.6 & 8.6 & 21.0 & 21.0 & 99 \\
\hline & $\Delta=40 \mathrm{~mm}$ & 67.0 & 122.0 & 100.0 & 8.1 & 40.8 & 41.0 & 30 \\
\hline & $\Delta=60 \mathrm{~mm}$ & 82.0 & 138.0 & 115.0 & 10.0 & 60.9 & 61.0 & 15 \\
\hline & $\Delta=80 \mathrm{~mm}$ & 88.0 & 141.0 & 121.0 & 11.2 & 80.9 & 80.8 & 10 \\
\hline \multirow{5}{*}{ 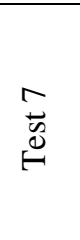 } & ECCS & 75.0 & 133.0 & 108.0 & 8.8 & 75.6 & 75.5 & 17 \\
\hline & $\Delta=20 \mathrm{~mm}$ & 61.0 & 118.0 & 87.0 & 5.7 & 20.8 & 21.1 & 127 \\
\hline & $\Delta=40 \mathrm{~mm}$ & 64.0 & 132.0 & 102.0 & 6.1 & 40.9 & 40.9 & 30 \\
\hline & $\Delta=60 \mathrm{~mm}$ & 81.0 & 141.0 & 115.0 & 8.8 & 60.7 & 60.8 & 12 \\
\hline & $\Delta=80 \mathrm{~mm}$ & 84.0 & 144.0 & 123.0 & 9.4 & 80.8 & 80.9 & 10 \\
\hline
\end{tabular}


Table 6: Summary of the cyclic loading tests (continuation)

\begin{tabular}{|c|c|c|c|c|c|c|c|c|}
\hline $\begin{array}{c}\text { Test } \\
\text { ID }\end{array}$ & $\begin{array}{l}\text { Loading } \\
\text { Protocol }\end{array}$ & $\begin{array}{c}\text { Fy } \\
{[\mathrm{kN}]}\end{array}$ & $\begin{array}{c}\mathrm{F}_{\max } \\
\text { (Comp.) } \\
{[\mathrm{kN}]}\end{array}$ & $\begin{array}{c}\mathrm{F}_{\max } \\
\text { (Tension) } \\
{[\mathrm{kN}]}\end{array}$ & $\begin{array}{c}\mathrm{u}_{\mathrm{y}} \\
{[\mathrm{mm}]}\end{array}$ & $\begin{array}{c}\mathrm{u}_{\max } \\
(\text { Comp.) } \\
{[\mathrm{mm}]}\end{array}$ & $\begin{array}{c}\mathrm{u}_{\max } \\
\text { (Tension) } \\
{[\mathrm{mm}]}\end{array}$ & $\begin{array}{l}\text { Total Number of } \\
\text { Cycles to Failure }\end{array}$ \\
\hline \multirow{5}{*}{ 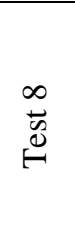 } & ECCS & 127.0 & 350.0 & 191.0 & 6.5 & 60.4 & 57.7 & 12 \\
\hline & $\Delta=20 \mathrm{~mm}$ & 122.0 & 253.0 & 154.0 & 6.4 & 20.5 & 20.8 & 53 \\
\hline & $\Delta=40 \mathrm{~mm}$ & 124.0 & 278.0 & 184.0 & 6.2 & 40.5 & 40.6 & 14 \\
\hline & $\Delta=60 \mathrm{~mm}$ & 134.0 & 374.0 & 213.0 & 7.9 & 60.3 & 60.3 & 7 \\
\hline & $\Delta=80 \mathrm{~mm}$ & 133.0 & 387.0 & 242.0 & 7.4 & 80.4 & 80.4 & 6 \\
\hline \multirow{5}{*}{$\underset{\vec{w}}{\stackrel{a}{\oplus}}$} & ECCS & 255.0 & 362.0 & 209.0 & 6.1 & 60.9 & 51.9 & 12 \\
\hline & $\Delta=20 \mathrm{~mm}$ & 124.0 & 273.0 & 156.0 & 6.1 & 20.6 & 20.5 & 68 \\
\hline & $\Delta=40 \mathrm{~mm}$ & 124.0 & 313.0 & 196.0 & 5.9 & 40.4 & 40.5 & 14 \\
\hline & $\Delta=60 \mathrm{~mm}$ & 110.0 & 374.0 & 190.0 & 5.8 & 60.2 & 40.2 & 5 \\
\hline & $\Delta=80 \mathrm{~mm}$ & 123.0 & 365.0 & 249.0 & 7.1 & 80.5 & 80.4 & 5 \\
\hline \multirow{5}{*}{ 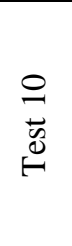 } & ECCS & 144.0 & 345.0 & 186.0 & 9.1 & 60.8 & 60.5 & 13 \\
\hline & $\Delta=20 \mathrm{~mm}$ & 115.0 & 278.0 & 158.0 & 5.5 & 20.6 & 20.8 & 62 \\
\hline & $\Delta=40 \mathrm{~mm}$ & 122.0 & 325.0 & 190.0 & 5.9 & 40.4 & 40.8 & 13 \\
\hline & $\Delta=60 \mathrm{~mm}$ & 122.0 & 364.0 & 219.0 & 6.0 & 60.6 & 60.5 & 7 \\
\hline & $\Delta=80 \mathrm{~mm}$ & 124.0 & 384.0 & 270.0 & 6.4 & 80.3 & 80.4 & 6 \\
\hline \multirow{5}{*}{ 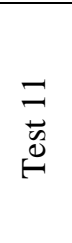 } & ECCS & 199.0 & 514.0 & 291.0 & 7.6 & 60.2 & 45.5 & 12 \\
\hline & $\Delta=20 \mathrm{~mm}$ & 179.0 & 415.0 & 223.0 & 6.2 & 20.4 & 20.7 & 55 \\
\hline & $\Delta=40 \mathrm{~mm}$ & 193.0 & 466.0 & 294.0 & 6.6 & 40.4 & 40.4 & 11 \\
\hline & $\Delta=60 \mathrm{~mm}$ & - & - & - & - & - & - & - \\
\hline & $\Delta=80 \mathrm{~mm}$ & 187.0 & 533.0 & 280.0 & 8.9 & 80.4 & 61.9 & 5 \\
\hline
\end{tabular}

Table 7: Cumulative absorbed energy

\begin{tabular}{|c|c|c|c|c|c|}
\hline Test ID & ECCS [kNm] & $\begin{array}{c}\Delta=20 \mathrm{~mm} \\
{[\mathrm{kNm}]}\end{array}$ & $\begin{array}{c}\Delta=40 \mathrm{~mm} \\
{[\mathrm{kNm}]}\end{array}$ & $\begin{array}{c}\Delta=60 \mathrm{~mm} \\
{[\mathrm{kNm}]}\end{array}$ & $\begin{array}{c}\Delta=80 \mathrm{~mm} \\
{[\mathrm{kNm}]}\end{array}$ \\
\hline Test 1 & 140 & 255 & 160 & 125 & 95 \\
\hline Test 2 & 155 & 300 & 162 & 230 & 252 \\
\hline Test 3 & 195 & 280 & 119 & 130 & 92 \\
\hline Test 4 & 145 & 252 & 240 & 182 & 132 \\
\hline Test 5 & 139 & 348 & 225 & 180 & 108 \\
\hline Test 6 & 150 & 248 & 198 & 140 & 125 \\
\hline Test 7 & 138 & 299 & 201 & 120 & 90 \\
\hline Test 8 & 98 & 215 & 122 & 85 & 47 \\
\hline Test 9 & 105 & 248 & 130 & 4.3 & 85 \\
\hline Test 10 & 115 & 248 & 120 & 85 & 40 \\
\hline Test 11 & 140 & 305 & 145 & - & \\
\hline
\end{tabular}

\section{Experimental tests on CBFs incorporating U-connection}

\subsection{General}

In order to assess the behavior of CBFs incorporating the U-connection, a group of experimental tests on large-scale CBFs with diagonal bracings was performed at the Laboratorio di Prove e Materiali of Politecnico di Milano. These tests were also executed 
within the scope of the INERD research project (INERD, 2004). The experimental tests consisted of cyclic loading tests with a horizontal load applied at the top of the frame. The test specimens were designed so that the whole frame and connection plates remain elastic and that plastic deformations only occur in the U-plate. In the following sections is presented a summary of this experimental testing programme and the obtained results.

\subsection{Test specimens, test programme and monitoring}

The configuration and global geometric dimensions of the large scale CBFs with diagonal bracing are depicted in Figure 10. In these frames, the connection between the bracing and the adjacent columns was achieved using the U-plates. Within the tests, the brace-tocolumn connection configuration was varied in order to cover both loading conditions of the U-plate, parallel and transverse (check §3.2), as in the experimental programme on isolated U-connections. In Figure 11, the connection configurations between braces and columns are detailed. As referred above, the test specimens were designed so that failure would occur in the U-plate (dissipative connection). The testing variables considered the same geometric properties of the plates as for the tests on the isolated connections (see Figure 4). In Table 8 and in Table 9 are summarized the main geometric and material properties of the tested specimens, respectively.

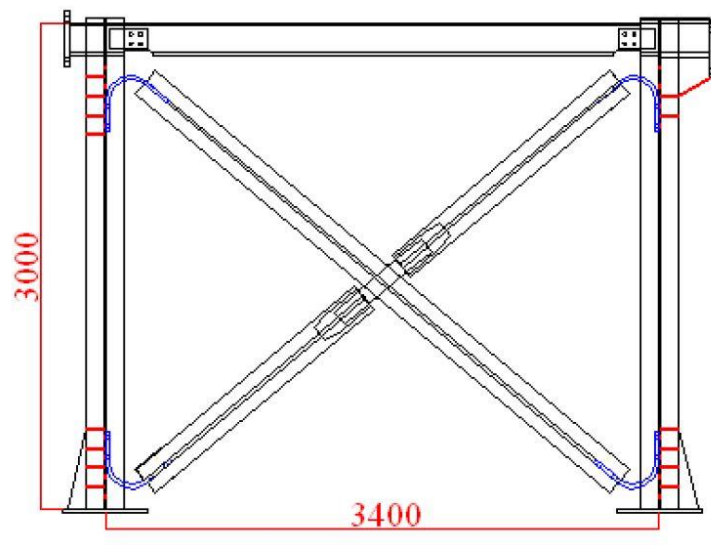

a) U-plate subject to parallel loading

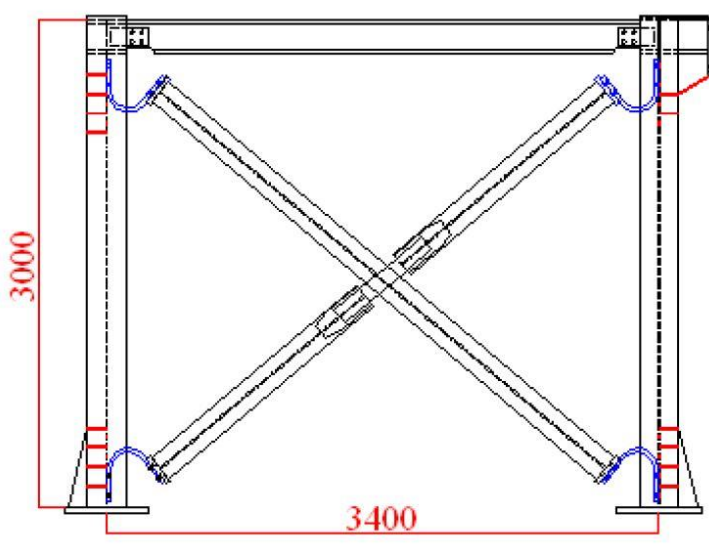

b) U-plate subject to transverse loading

Figure 10: Global geometry of the frame test specimens 


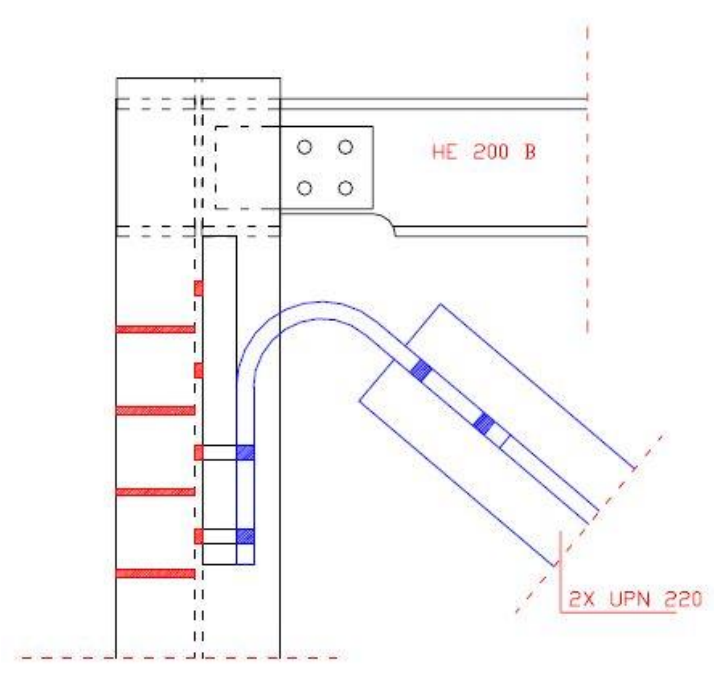

a) U-plate subject to parallel loading

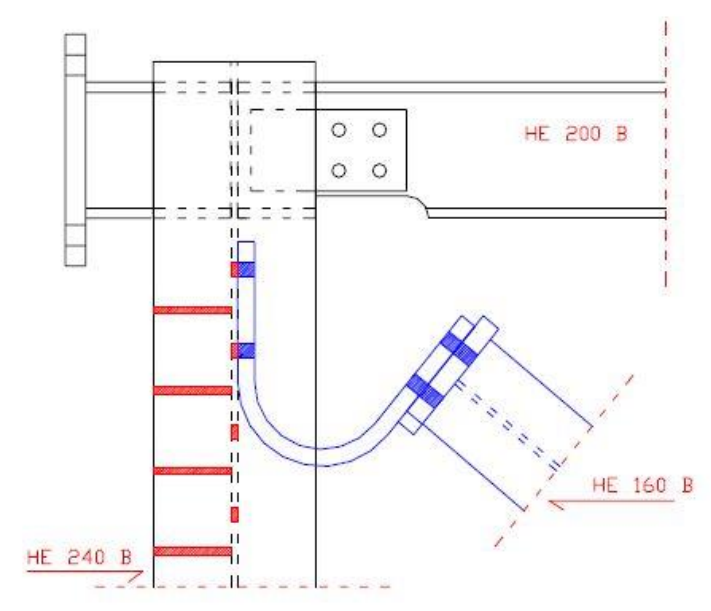

b) U-plate subject to transverse loading

Figure 11: Test specimens detail of the brace-column connection using U-plate

Table 8: Summary of the geometric properties of the test specimens (U-plate) and range of variation in the frame tests

\begin{tabular}{|c|c|c|c|c|c|c|c|c|}
\hline $\mathbf{R}[\mathbf{m m}]$ & $\begin{array}{c}\boldsymbol{\alpha} \\
{\left[{ }^{\mathbf{0}}\right]}\end{array}$ & $\begin{array}{c}\mathbf{e} \\
{[\mathbf{m m}]}\end{array}$ & $\begin{array}{c}\mathbf{b} \\
{[\mathbf{m m}]}\end{array}$ & $\begin{array}{c}\mathbf{h} \\
{[\mathbf{m m}]}\end{array}$ & $\begin{array}{c}\mathbf{e}_{1} \\
{[\mathbf{m m}]}\end{array}$ & $\begin{array}{c}\mathbf{e}_{2} \\
{[\mathbf{m m}]}\end{array}$ & $\begin{array}{c}\mathbf{p}_{1} \\
{[\mathbf{m m}]}\end{array}$ & $\begin{array}{c}\mathbf{p}_{2} \\
{[\mathbf{m m}]}\end{array}$ \\
\hline $100 ; 125$ & $40 ; 50$ & $25 ; 30$ & 160 & 205 & 40.5 & 40 & 120 & 80 \\
\hline
\end{tabular}

Table 9: Mechanical properties of the test specimens materials in the frame tests

\begin{tabular}{|c|c|c|}
\hline \multicolumn{2}{|c|}{ Steel Plates (U-plates) } & Bolts \\
\hline \multicolumn{2}{|c|}{ S355 } & \multirow{6}{*}{$\begin{array}{l}\text { M20-8.8 } \\
\text { M27 -8.8 } \\
\text { M20-10.9 } \\
\text { M27-10.9 }\end{array}$} \\
\hline \multicolumn{2}{|c|}{ Coupon Tests: } & \\
\hline $\mathrm{t}=25 \mathrm{~mm}$ & $\mathrm{t}=30 \mathrm{~mm}$ & \\
\hline $\mathrm{f}_{\mathrm{y}}=381 \mathrm{~N} / \mathrm{mm}^{2}$ & $\mathrm{f}_{\mathrm{y}}=347 \mathrm{~N} / \mathrm{mm}^{2}$ & \\
\hline $\mathrm{f}_{\mathrm{u}}=540 \mathrm{~N} / \mathrm{mm}^{2}$ & $\mathrm{f}_{\mathrm{u}}=529 \mathrm{~N} / \mathrm{mm}^{2}$ & \\
\hline$\varepsilon_{\mathrm{u}}=32.9 \%$ & $\varepsilon_{\mathrm{u}}=30.9 \%$ & \\
\hline
\end{tabular}

The testing programme contemplated the execution of cyclic tests following the ECCS cyclic loading protocol (ECCS, 1986). The loading was applied by an actuator at the top of the column in displacement control mode. In Table 10 is summarized the full experimental programme identifying the test variables. The cyclic loading protocol consisted in starting with small cycle amplitudes increased up to the identification of the elastic deformation limit. From this point on, the ECCS cyclic loading protocol (ECCS, 1986) was followed on the basis of the identified yield deformation. The test data acquisition system consisted in: i) actuator load cell, for the load applied to the frame; ii) displacement transducers, for frame deformation and axial deformation of the bracings; iii) strain gauges. 
Table 10: Test programme on CBF incorporating the U-plate

\begin{tabular}{|c|c|c|c|c|c|}
\hline Test ID & $\mathbf{R}[\mathbf{m m}]$ & e $[\mathrm{mm}]$ & $\alpha\left[^{0}\right]$ & Position & $\mathrm{N}^{\circ}$ of tests \\
\hline CBF 1 & 125 & 25 & 50 & \multirow{4}{*}{$\begin{array}{l}\text { एा1 } \\
\text { Parallel } \\
\text { Loading }\end{array}$} & \multirow{8}{*}{$\begin{array}{l}1 \text { cyclic quasi- } \\
\text { static } \\
\text { according to } \\
\text { the ECCS } \\
\text { cyclic loading } \\
\text { protocol } \\
\text { (ECCS, 1986) }\end{array}$} \\
\hline CBF 2 & 100 & 25 & 50 & & \\
\hline CBF 3 & 125 & 30 & 50 & & \\
\hline CBF 4 & 100 & 30 & 50 & & \\
\hline CBF 5 & 100 & 30 & 40 & \multirow{4}{*}{ 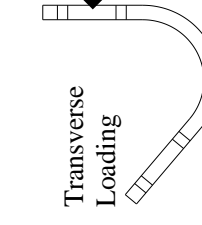 } & \\
\hline CBF 6 & 100 & 25 & 40 & & \\
\hline CBF 7 & 125 & 30 & 40 & & \\
\hline CBF 8 & 125 & 25 & 40 & & \\
\hline
\end{tabular}

\subsection{Summary of the test results}

The tests executed on global frames allowed extracting force-deformation curves (cyclic loops) both for the global frame and for the U-connections. In Figure 12 are presented the force-interstorey drift of test specimens CBF 1 and CBF 8. These test specimens incorporate the U-connection with parallel loading and transverse loading, respectively. The U-plate geometric dimensions differ only through the bent angle, $50^{\circ}$ and $40^{\circ}$, respectively. Given the limitations on the geometric dimensions of the frame due test layout, it was not possible to reach exactly the same angle for the two types of loading direction of the U-plate. The obtained response shows that, despite the asymmetric response of the U-plate (check Figure 8), the global response is as expected symmetric since the frame is also symmetric; in each direction, two connections work in tension and another two in compression. The frame incorporating the U-plate with transverse load (CBF 8) presents slightly higher load and deformation capacity. In Table 11 are summarized the results of tests on the CBFs. It is clear that for the frames where the Uplate is loaded with transverse loading, the frame stiffness and load capacity are higher. This is in agreement with the results of the test on isolated U-connections (remember Table 4). In terms of deformation capacity (maximum deformation) and number of cycles to failure, the different configurations present very similar results. This observation is however not really relevant, given that, due to limitations on the experimental testing system to accommodate very large displacement, the tests were interrupted before the complete failure of the connections. In terms of absorbed energy, frames with transverse loading of the U-plate present again a higher cumulative absorbed energy. This results from the fact that these frames present higher resistance, but at the same time have a similar deformation capacity as the frames with U-plate loaded in the parallel direction. 


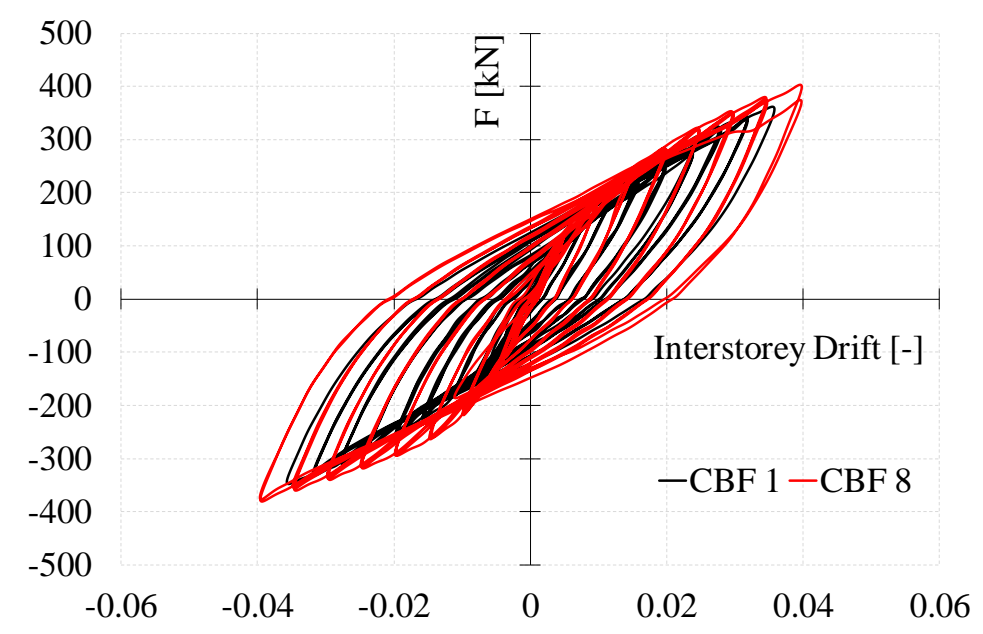

Figure 12: Global frame response

Table 11: Summary of cyclic loading tests on CBF incorporating U-plate

\begin{tabular}{|c|c|c|c|c|c|c|c|c|c|}
\hline \multirow[b]{2}{*}{ Test ID } & \multirow{2}{*}{$\begin{array}{c}\mathrm{Ky} \\
{[\mathrm{kN} / \mathrm{mm}]}\end{array}$} & \multirow[b]{2}{*}{$\begin{array}{c}\mathrm{F}_{\mathrm{y}} \\
{[\mathrm{kN}]}\end{array}$} & \multirow[b]{2}{*}{$\begin{array}{c}\mathrm{u}_{\mathrm{y}} \\
{[\mathrm{mm}]}\end{array}$} & \multicolumn{2}{|c|}{ Dir. $\rightarrow$} & \multicolumn{2}{|c|}{ Dir. $\leftarrow$} & \multirow{2}{*}{$\begin{array}{c}\text { Cum. } \\
\text { Engergy } \\
{[\mathrm{kN} . \mathrm{m}]}\end{array}$} & \multirow[b]{2}{*}{$\mathrm{N}^{\mathrm{o}}$ of Cycles } \\
\hline & & & & $\begin{array}{l}\mathrm{F}_{\max } \\
{[\mathrm{kN}]}\end{array}$ & $\begin{array}{c}\mathrm{u}_{\max } \\
{[\mathrm{mm}]}\end{array}$ & $\begin{array}{l}\mathrm{F}_{\max } \\
{[\mathrm{kN}]}\end{array}$ & $\begin{array}{c}\mathrm{u}_{\max } \\
{[\mathrm{mm}]}\end{array}$ & & \\
\hline CBF 1 & 6.93 & 210.0 & 30.30 & 360.10 & 119.40 & 372.21 & 119.07 & 550 & 33 \\
\hline CBF 2 & 9.20 & 215.0 & 23.40 & 400.9 & 119.40 & 404.83 & 119.11 & 470 & 25 \\
\hline CBF 3 & 8.83 & 257.0 & 29.10 & 425.10 & 118.80 & 461.57 & 117.59 & 450 & 26 \\
\hline CBF 4 & 11.33 & 298.0 & 26.30 & 479.40 & 119.90 & 514.00 & 119.30 & 650 & 27 \\
\hline CBF 5 & 15.02 & 395.0 & 26.30 & 514.80 & 104.60 & 527.08 & 104.68 & 750 & 24 \\
\hline CBF 6 & 12.76 & 273.0 & 21.40 & 403.20 & 118.60 & 416.41 & 104.44 & 640 & 25 \\
\hline CBF 7 & 12.55 & 340.0 & 27.10 & 479.90 & 119.20 & 495.03 & 119.43 & 610 & 26 \\
\hline CBF 8 & 10.15 & 209.0 & 20.60 & 380.80 & 119.00 & 403.01 & 119.39 & 540 & 27 \\
\hline
\end{tabular}

Figure 13 shows the final deformation state of the test specimens CBF 1 and CBF 8. The test on specimen CBF 1 was interrupted not because of a fracture in the material but due to significant plastic deformation/damage of the U-plate (Figure 13-a). In test specimen CBF 8, the test was interrupted due to fracture in the net-section of the U-plate in the row of bolts with higher load. Nevertheless, significant plastic deformation was still observed in the U-plate (Figure 13-b). Figure 13-a) highlights the plastic deformations developed in the curved surface of the U-plate (lighter color of the steel surface). In Figure 13-b), it is possible to observe that plastic deformation also occurs at the edge of the end plate of the bracing. This occurs when the U-plate is compressed by the brace, because the Uplate is clamped to the end-plate at the edge of the brace. As it can be observed, the latter is very stiff and behaves then as a rigid element. 

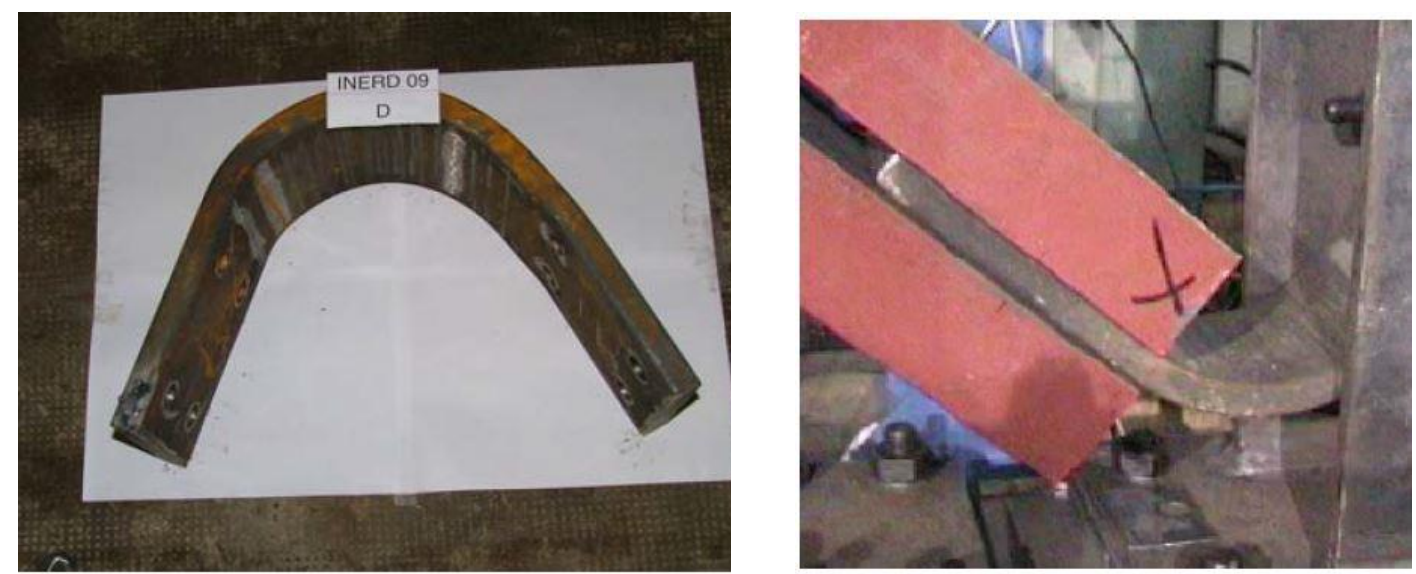

a) $\mathrm{CBF} 1$
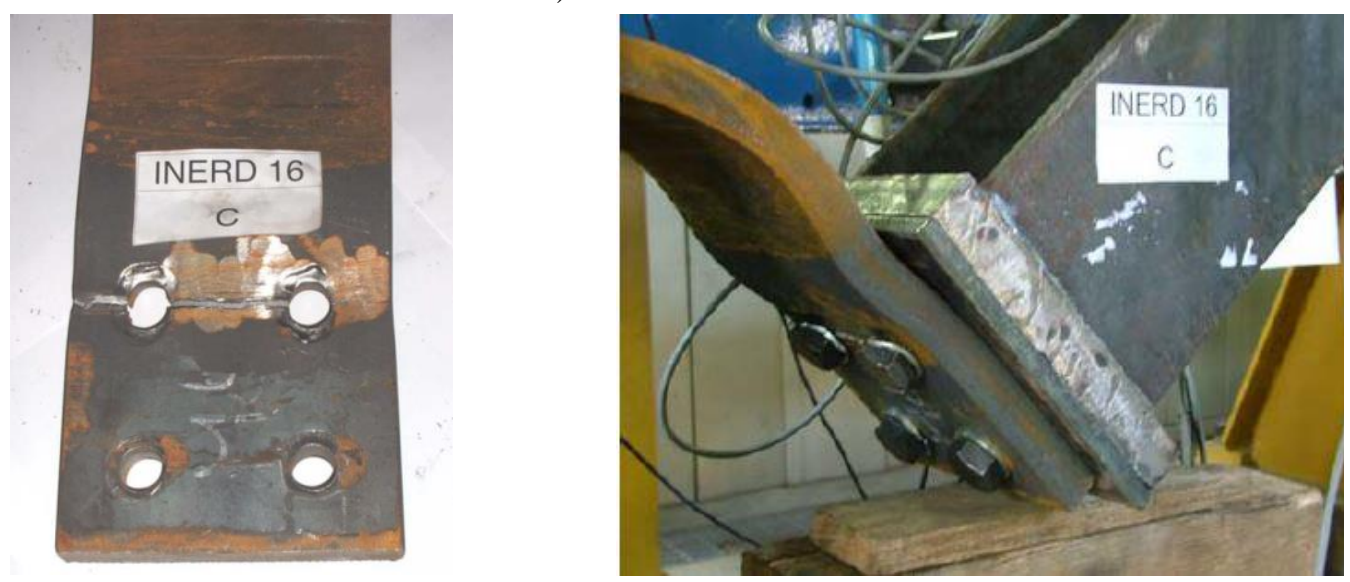

b) $\mathrm{CBF} 8$

Figure 13: Final deformation of the U-connection after the cyclic tests on Frames

\section{Analysis of the experimental results}

\subsection{Tests on isolated U-connections}

In Figure 14 a comparison between the different test specimens under monotonic loading is presented. Figure 14-a) shows the results for the monotonic tests with compression loading and Figure 14-b) the results of the tests with tension loading. The five mechanical properties, described in $\S 3$, are used:

- $F_{y}$ - force at elastic limit;

- $S$ - initial stiffness;

- $u_{\max }$ - maximum deformation;

- $F_{\max }$ - maximum force;

- P. Energy - plastic energy (resulting from inelastic deformations).

In order to allow a rational global assessment, in Figure 14 the values of the referred properties are presented in a normalized format: for each parameter, the ratio between the value obtained for the test specimen $i$ and the maximum value obtained for the full set of test specimens is given. In general, except for the maximum deformation, the U-plate subject to a transverse load presents higher ratios for both type of loading (i.e. compression and tension). Though the U-plate with transverse loading has lower 
deformation capacity, high plastic energy is still observed. For this loading condition, the lower deformation capacity is compensated by a higher load capacity. Consequently, a good dissipation behavior can be obtained independently of the load position.

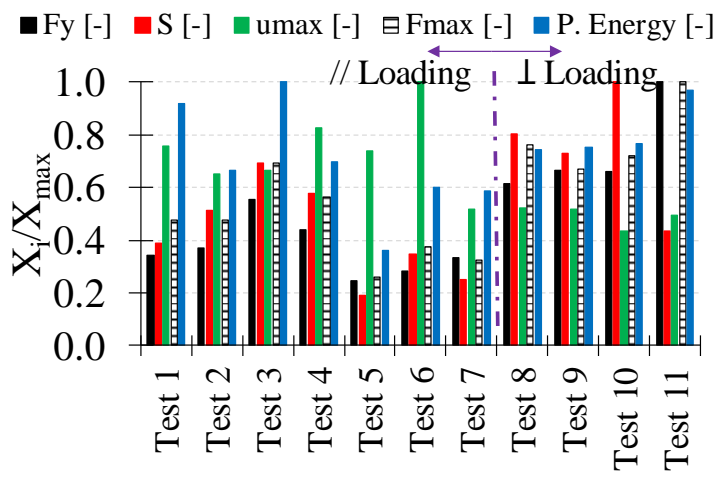

a) Compression loading

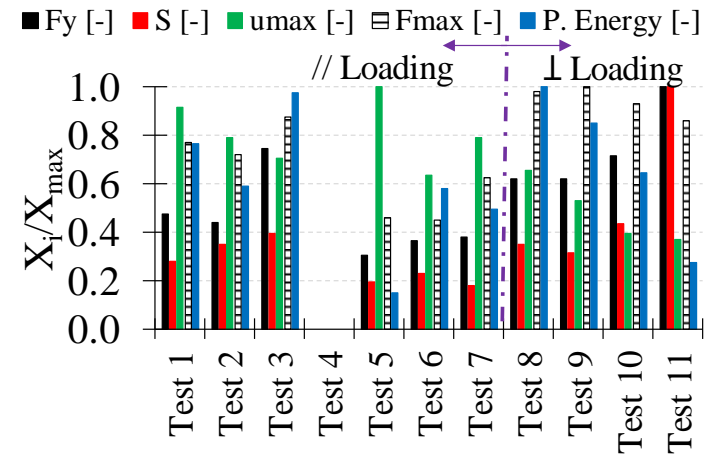

b ) Tension loading

Figure 14: Comparison of the U-plate performance for the different monitoring parameters in the monotonic tests

In Figure 15, a comparison between the loading type, compression and tension, is presented. For each of the above referred mechanical properties and for the two types of loading, the average of all test specimens was computed. Here, no differentiation between the loading orientation is made. In the vertical axis, the ratio between the average for each loading type and the maximum average is represented. The U-plate loaded in compression presents higher values for all considered mechanical properties. The reason of these results is explained by the fact that when loaded in compression, the U-plate is pushed against the adjacent member. In this case the testing frame structure, provides some additional restraint, increasing load capacity and stiffness. In a real frame, this effect will also be provided by the connected members: the bracings and the beam/column.

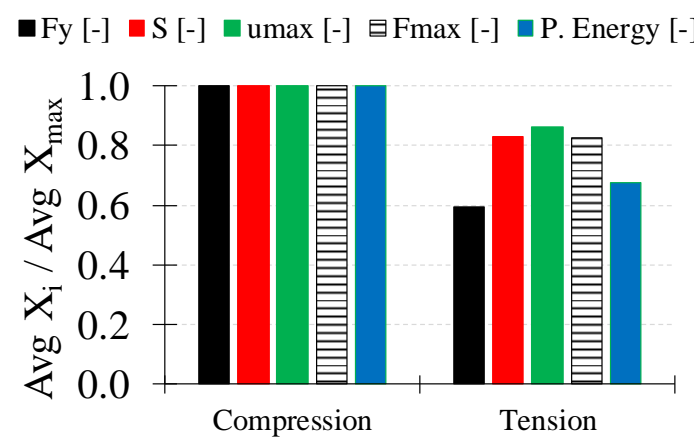

Figure 15: Comparison between the performance of the U-connection in compression and in tension loading

In Figure 16 the influence of each geometric parameter, varied within the experimental programme, is shown. For each variable, two charts are given, one for compression loading and one for tension loading. The ratio between test results of each test specimen and a reference test specimen was computed for the mechanical properties referred above $\left(F_{y}, S, u_{\max }, F_{\max }, P\right.$. Energy). According to the variable under analysis, the following reference test specimens were used: i) $t=25 \mathrm{~mm}$ (thickness); ii) $\alpha=45^{\circ}$ (bent angle); iii) 
$R=100 \mathrm{~mm}$ (bent radius). In order to isolate the variable under analysis, the ratio was calculated between test specimens where the other geometric properties are kept constant. In Figure 16 a) and b), the results regarding the variation of the U-plate thickness are presented. Note that to above referred problems in the execution of Test 4 subjected to tension loading, no result is presented for the case $\left(\alpha=50^{\circ} ; \mathrm{R}=125 \mathrm{~mm}\right.$; / $)$. For both loading directions, the overall trend is that the value of the mechanical properties will increase with of the U-plate thickness. This is achieved for all cases for the force at elastic limit $\left(F_{y}\right)$ and the initial stiffness $(S)$. For the other mechanical properties ( $u_{\max }, F_{\max }, P$. Energy), a difference is observed between the results according to the load direction. In compression, the increase of plate thickness also results in an increase of these mechanical properties. In tension, an exception is mainly observed for a bent angle of $39^{\circ}$. For this case, a decrease is notice in all these mechanical properties. Given the dependency of plastic energy on the maximum deformation $\left(u_{\max }\right)$ and maximum force $\left(F_{\max }\right)$, the variation on the two latter parameters is consequently reflected in the first.

In Figure 16 c) and d), the results reflecting the influence of the angle of the U-plate are depicted. For this geometric property there is no clear trend, depending on the other geometric properties of the U-plate the variation of the bent angle will result in an increase or decrease of the different mechanical properties. Nevertheless, if one wants to take a general conclusion, based on the majority of the computed ratios, it can state that the variation of the bent angle will result in a reduction of the mechanical properties, except for the maximum deformation $\left(u_{\max }\right)$. For the latter, the results are in the opposite direction.

In Figure 16 e) and $\mathrm{f}$ ), the impact of the bent radius of the U-plate is shown. It is clear that increasing the bend radius from $100 \mathrm{~mm}$ to $125 \mathrm{~mm}$ results in a general decrease of the mechanical performances. The main exception is the maximum deformation capacity when loaded in compression.

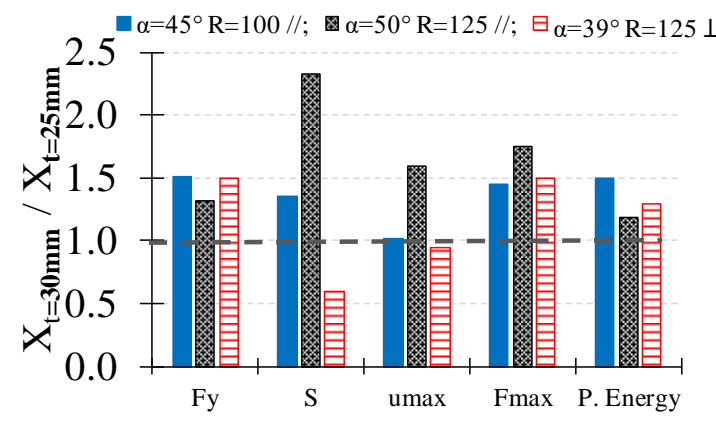

a) Compression loading (thickness)

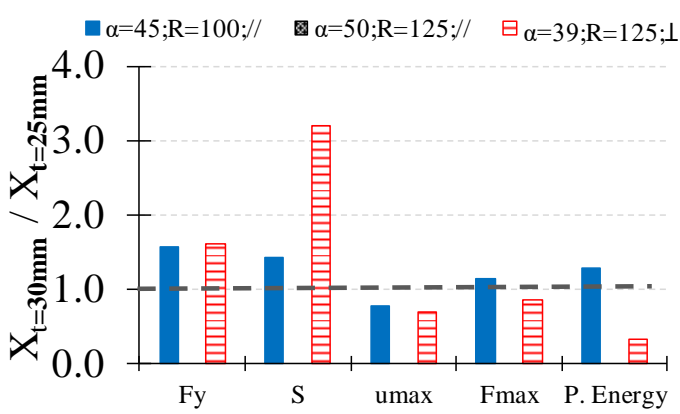

b) Tension loading (thickness) 


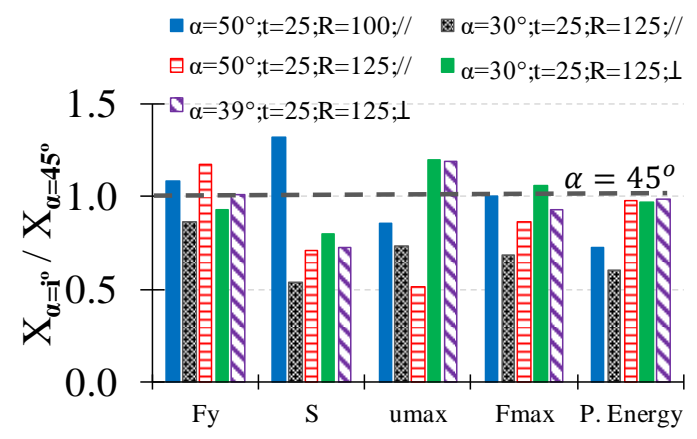

c) Compression loading (bent angle)

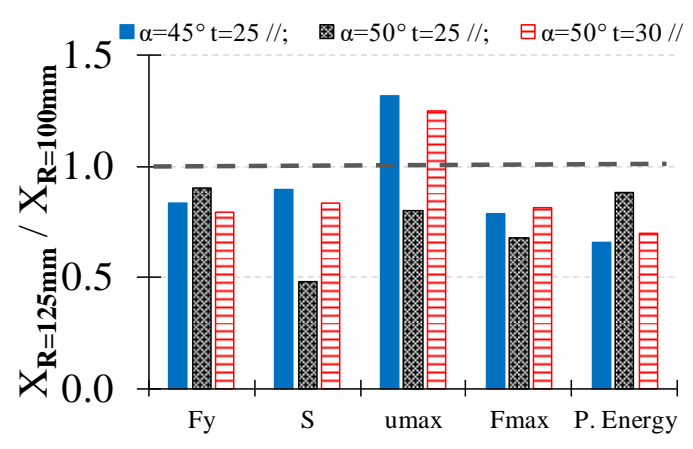

e) Compression loading (bent radius)

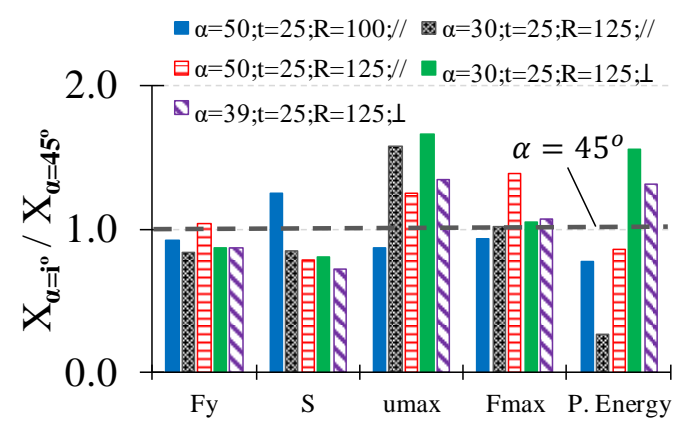

d) Tension loading (bent angle)

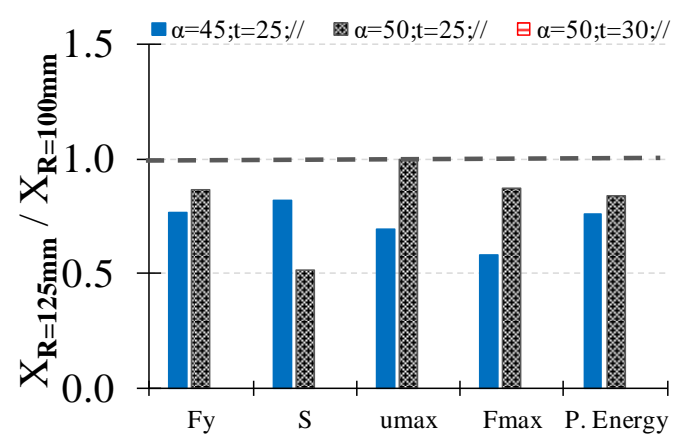

f) Tension loading (bent radius)

Figure 16: Influence of the different geometric variations on the performance of the U-connection

In order to evaluate the performance of the U-plate under cyclic loading, the accumulated energy and the number of completed cycles are used. In Figure 17 are summarized the results of the cyclic tests, for the referred parameters, considering all test specimens configurations. In Figure 17-a) are presented only the results of the cyclic tests using the ECCS cyclic protocol (ECCS, 1986). In Figure 17-b) are shown the results for all the other cyclic tests on isolated connections, i.e. the tests performed under constant cycling amplitude up to failure. In Figure 17-a) can be observed that specimen 3 is the U-plate configuration presenting the highest energy dissipation capacity. In this test specimen, the load was applied parallel to the U-plate legs. The graph also shows that the maximum energy dissipation is not related to the largest number of cycles, which is obtained for Test 5. Then, it is also evident that the U-plates with parallel loading present higher energy dissipation than the test specimens with transverse loading. For the other cyclic tests (Figure 17-b) the same general conclusions can be drawn. What is clearly evident is the fact that with increasing cycling amplitude, the U-plate loses energy dissipation capacity as the number of imposed cycles decreases increases. These results demonstrate that this type of connection is sensitive to cyclic loading and therefore prone to low cycle fatigue. 


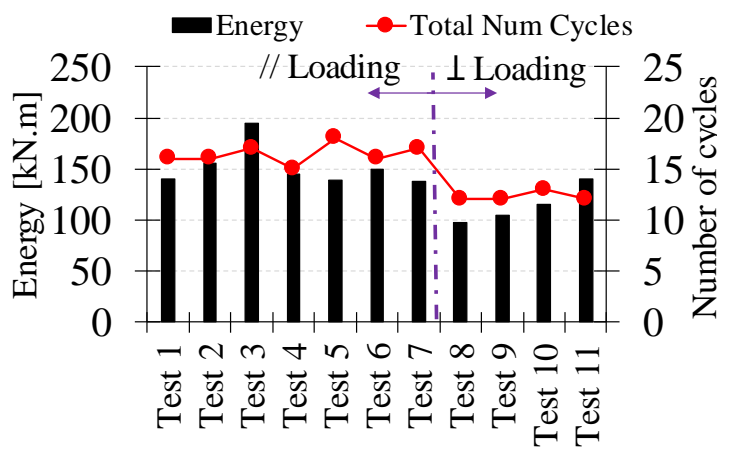

a) ECCS cyclic loading protocol

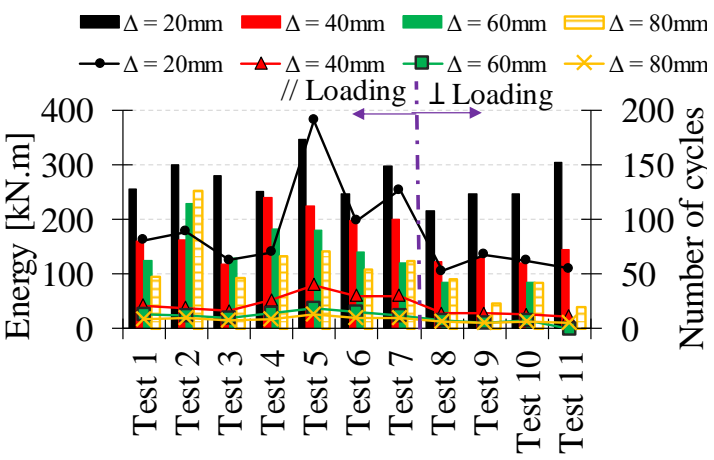

b) Cyclic loading with constant amplitude

Figure 17: Comparison of the performance of the U-plate in the cyclic tests

Figure 18, as in Figure 16, shows the influence of the different geometrical variations. All cyclic tests are considered. A chart for the accumulated energy (left hand side) and for the number of cycles (right hand side) is drawn.

Figure 18 a) and b) shows the results for the thickness variation. In general terms, the increase of thickness results in an increase of accumulated energy and a decrease of the number of cycles to failure. The increase of thickness leads to an increase of resistance which compensates the loss of endurance.

Figure $18 \mathrm{c}$ ) and d) shows the results for the angle variation. For this geometric variation, the majority of the computed ratios are around 1 which means that the angle variation has a low impact on the cyclic performances.

Figure $18 \mathrm{e}$ ) and $\mathrm{f}$ ) present the results in terms of the radius of the U-plate. For the ECCS cyclic protocol, this geometric variation results in barely no change in the accumulated energy nor in the number of cycles to failure. The exception is the U-plate with a bent angle of $50^{\circ}$, thickness of $30 \mathrm{~mm}$ and loaded parallel, where a reduction of both the accumulated energy and of the number of cycles to failure is observed. For the other cyclic tests, in particular for the lower amplitudes, increasing the bent radius results in an increase of both accumulated energy and number of cycles.

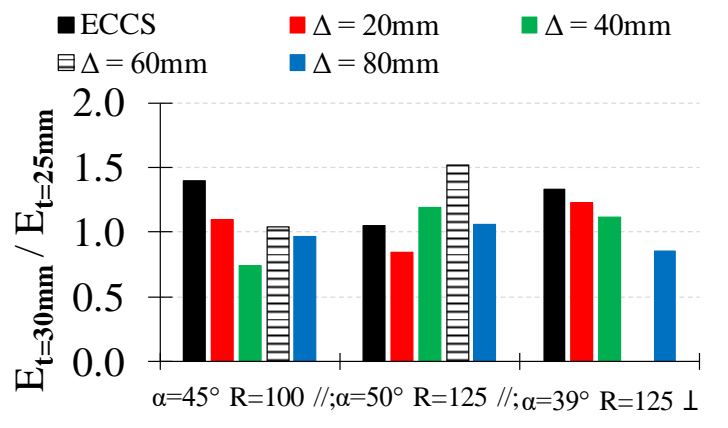

a) Thickness, Energy

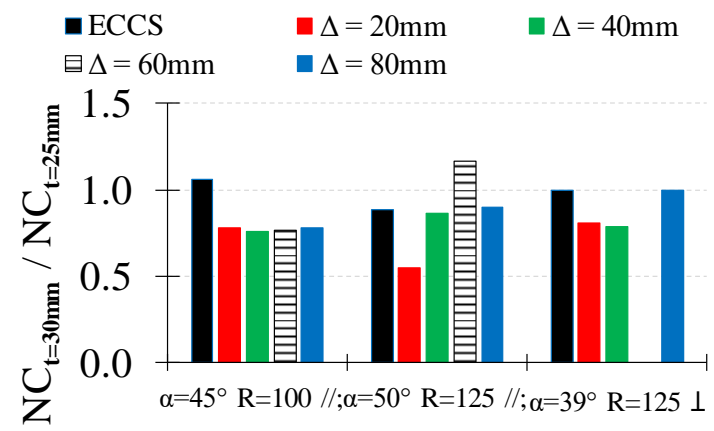

b) Thickness, Number of cycles 


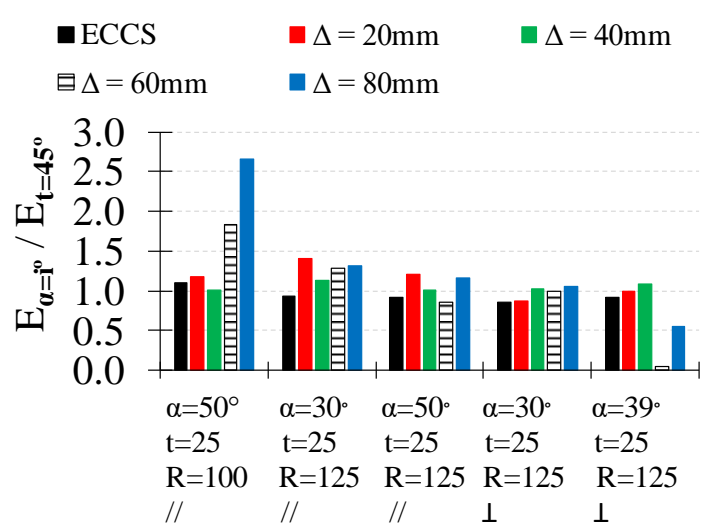

c) Bent angle, Energy

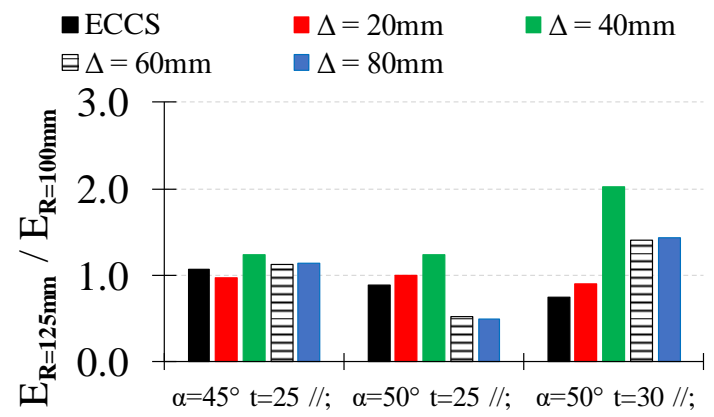

e) Bent radius, Energy

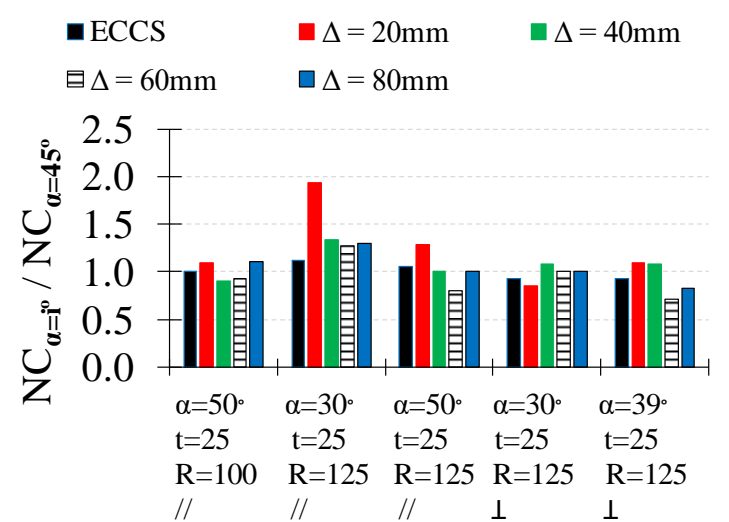

d) Bent angle, Number of cycles

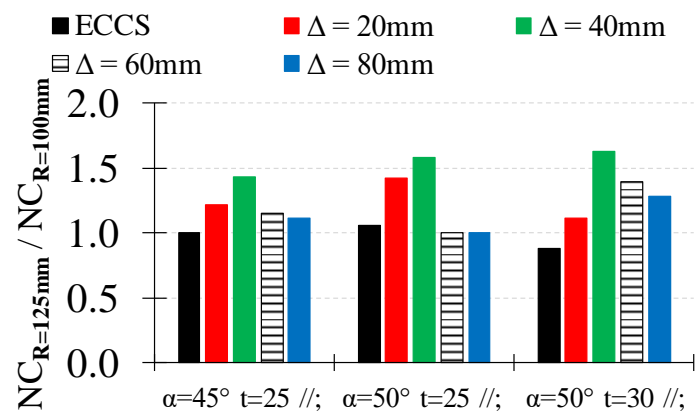

f) Bent radius, Number of cycles

Figure 18: Influence of the different geometric variations on the performance of the U-connection under cyclic loading

Figure 19 shows a comparison of the test results in monotonic loading with tests in cyclic loading, illustrating the low cyclic fatigue effect in the U-plate. The plotter parameter is the deformation at failure. The ratio between cyclic and monotonic loading was computed and is presented in the vertical axis. On the horizontal axis each test specimen is presented. Two ratios were computed: one using the monotonic compression loading and the other using the monotonic tension loading. For the cyclic tests, only the ECCS cyclic protocol was considered. It is clear that for all tested specimens, a reduction of the maximum deformation is obtained when the U-plate is subjected to a cyclic loading. This demonstrates that the U-plate is sensitive to cyclic loading therefore, possibly failing due to fatigue. The average reduction of deformation capacity is in the order of $50 \%$.

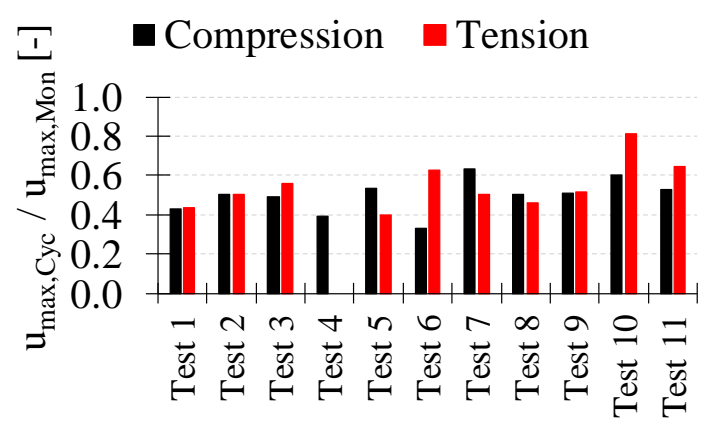


Figure 19: Comparison between the ultimate deformation of the U-plate under monotonic and cyclic loading (ECCS cyclic loading protocol)

\subsection{Tests on CBFs incorporating U-connection}

A detailed analysis of the results related to CBFs incorporating the U-connection is performed in the present section. In Figure 20 are compared all the test specimens. Figure 20-a) gives the results for the following mechanical properties:

- $F_{y}$ - lateral yield force;

- $S$ - initial lateral stiffness of the frame;

- $u_{\max }$ - maximum lateral deformation of the frame;

- $F_{\max }$ - maximum lateral force applied on the frame.

The ratio between a test specimen result and the maximum within all tests is used for the vertical axis. The chart shows equal maximum lateral deformation of all tests, which translates the limitation on the experimental testing system referred in $\S 4.3$, making the evaluation of this parameter less relevant than the others.

As for the tests on isolated connections, it is possible to observe that the frames with the U-connection subjected to a transverse loading exhibit better mechanical performance. Test 5 has the highest elastic limit force, highest maximum force and highest initial stiffness. This is consistent with the observations on the isolated U-connections tests where the U-connection with lower bent radius, higher thickness and subjected to a transverse loading presented the best mechanical properties. Figure 20-b) shows that the frames with transverse loading of the U-connection absorbed more energy. The higher dissipation of energy is mainly due to the higher resistance, since the maximum deformation capacity of the U-connection could not be attained within the majority of tests. The number of cycles performed does not varies considerably, and this is because the maximum deformation imposed to the U-connection (depending on the capacity of the test jack) was not sufficient to reach the complete failure of the specimen. The estimation of the dissipated energy and cycles at failure is thus a conservative approximation of the actual capacity.

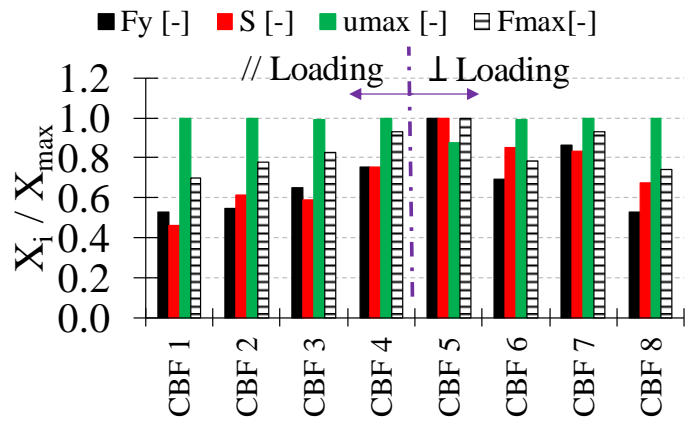

a) Frame mechanical properties

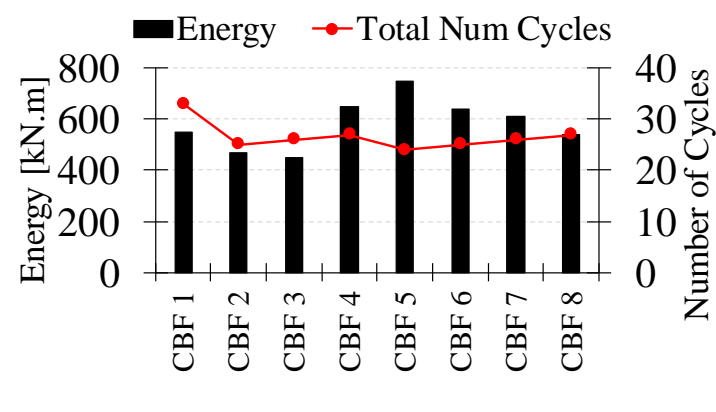

b) Energy and number of cycles

Figure 20: Comparison of the performance of all frame test specimens 
Figure 21 analyzes the different testing variables considered in the frame tests, namely the U-plate thickness and bent radius. As for the tests on isolated U-connections, the vertical axis gives the ratio between the testing variable and the reference variable. The horizontal axis identifies the geometric properties and loading direction. The parameters used for the analysis are the absorbed energy and the number of cycles to failure.

Figure 21-a) shows the impact of the variation of the plate thickness. Except for one geometry in a total of four, an increase of the plate thickness lead to an increase of absorbed energy. In what regards the number of cycles to achieve failure, the number was, in general, reduced. These results are in line with the results of the tests on the isolated connections (Figure 18- $\mathrm{a}$ and $\mathrm{b}$ ).

Figure 21-b) presents the results in terms of variation of the bent radius. Here it can be seen that the increase of the bent radius leads in general to a decrease of the absorbed energy and an increase of the number of cycles. This indicates that a plate with a radius of $125 \mathrm{~mm}$ is less stiff than with a radius of $100 \mathrm{~mm}$ but is on the other hand less sensitive to the degradation of the mechanical properties of the connection. Comparing Figure 21 with Figure 18, similar results can be observed on the impact of the geometric variations performed in both connection and frame tests. This indicates that the U-connection controlled also the behavior of the frames, as targeted by the design procedure of the CBFs test specimens.

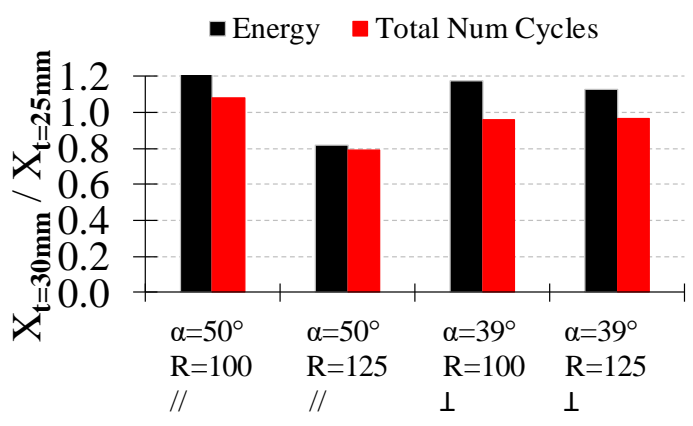

a) Thickness

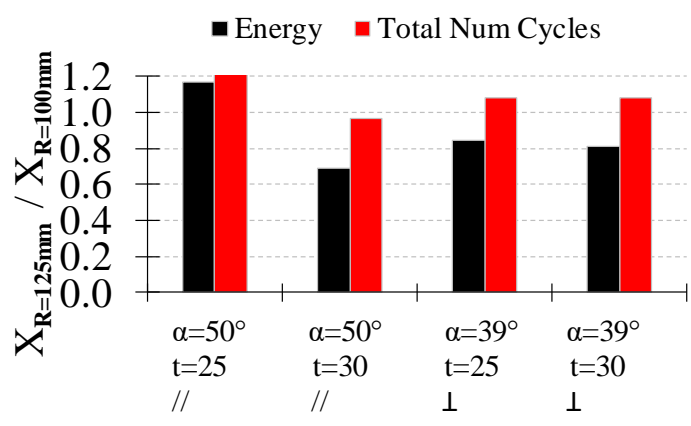

b) Bent radius

Figure 21: Influence of the different geometric variations on the performance of CBF incorporating the Uconnection under cyclic loading

\subsection{Comparative analysis: U-connection vs CBFs with Uconnection}

In order to relate the response of the tests on the isolated U-plate with the response of the CBFs incorporating the U-connection, a force-displacement response of the frames was extrapolated from the experimental results on the isolated connections. In Figure 22-a), the frame and its components are identified. The global response to lateral loading may be reproduced assuming a mechanical model with springs as given in Figure 22-b). In this model, each component of the $\mathrm{CBF}$ is represented by a extensional spring such as :

- Frame - Hinged Frame (without braces);

- $B_{i}$ - Diagonal Braces;

- $U_{i, j}-\mathrm{U}$-connections connecting braces to adjacent members. 
Consequently, the detailed model can be simplified through the assembly process, reducing first the springs in series to an equivalent spring (Assembly 1) and then parallel springs into an equivalent spring (Assembly 2). Table 12 gives the mathematical application of the described principle.

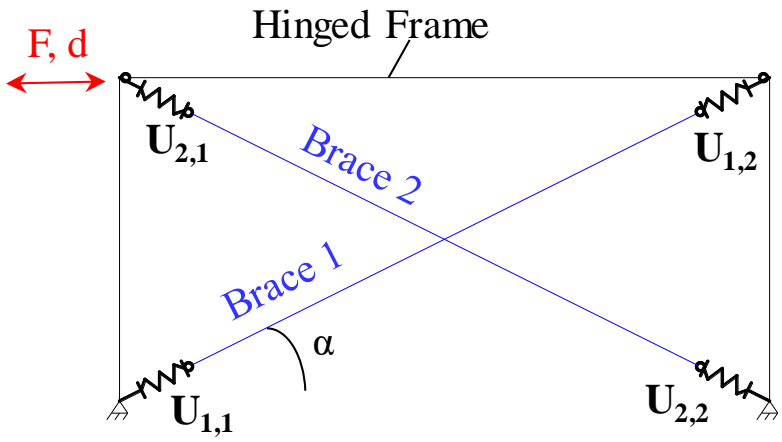

a) $\mathrm{CBF}$ and its components

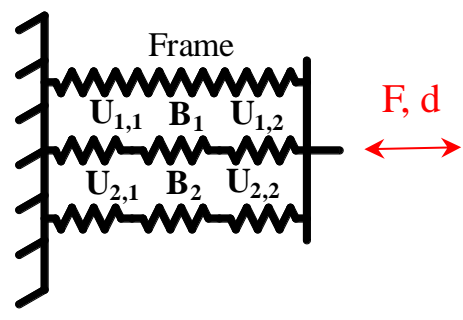

Detailed model

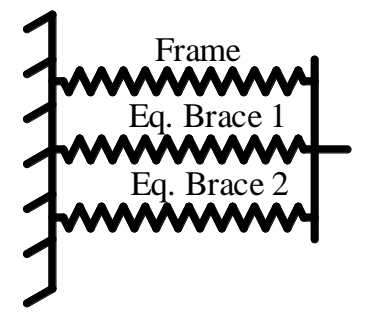

Assembly 1

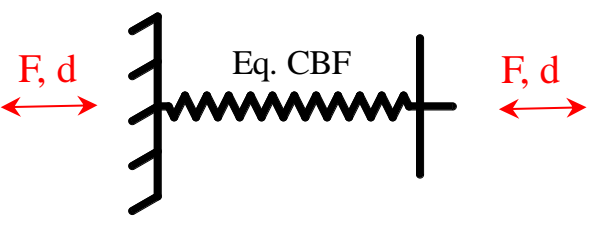

Assembly 2

b) Spring mechanical model

Figure 22: Mechanical model of CBF incorporating the U-connection for lateral loading

Table 12: Assembly of the spring mechanical model reproducing the CBF mechanical behaviour

\begin{tabular}{|c|c|c|}
\hline Model & Components & Mechanical Properties to Lateral Loading \\
\hline \multirow{3}{*}{ Detailed } & Frame & $\mathrm{F}_{\text {frame }}, \mathrm{d}_{\text {frame }}, \mathrm{K}_{\text {frame }}$ \\
\hline & Braces* & $\begin{array}{c}\mathrm{F}_{\mathrm{Bi}}, \mathrm{d}_{\mathrm{Bi}}, \mathrm{K}_{\mathrm{Bi}} \\
\text { with } \mathrm{i}=1 \rightarrow 2\end{array}$ \\
\hline & U-connections* & $\begin{array}{c}\mathrm{F}_{\mathrm{Ui}, \mathrm{j}}, \mathrm{d}_{\mathrm{Ui}, \mathrm{j}}, \mathrm{K}_{\mathrm{Ui}, \mathrm{j}} \\
\text { with } \mathrm{i}=1 \rightarrow 2 \text { and } \mathrm{j}=1 \rightarrow 2\end{array}$ \\
\hline \multirow{3}{*}{ Assembly 1} & Frame & $\mathrm{F}_{\text {frame }}, \mathrm{d}_{\text {frame }}, \mathrm{K}_{\text {frame }}$ \\
\hline & Eq. Brace 1 & $\begin{array}{c}F_{E q, B 1}=\operatorname{Min}\left(F_{U 1,1} ; F_{B 1} ; F_{U 1,2}\right) \\
d_{E q, B 1}=d_{U 1,1}+d_{B 1}+d_{U 1,2} \\
\frac{1}{K_{E q, B 1}}=\frac{1}{K_{U 1,1}}+\frac{1}{K_{B 1}}+\frac{1}{K_{U 1,2}}\end{array}$ \\
\hline & Eq. Brace 2 & $\begin{array}{c}F_{E q, B 2}=\operatorname{Min}\left(F_{U 2,1} ; F_{B 2} ; F_{U 2,2}\right) \\
d_{E q, B 2}=d_{U 2,1}+d_{B 2}+d_{U 2,2} \\
\frac{1}{K_{E q, B 2}}=\frac{1}{K_{U 2,1}}+\frac{1}{K_{B 2}}+\frac{1}{K_{U 2,2}}\end{array}$ \\
\hline Assembly 2 & Eq. CBF & $\begin{array}{c}F_{E q, C B F}=F_{\text {frame }}+F_{E q, B 1}+F_{E q, B 2} \\
d_{E q, C B F}=d_{\text {frame }}=d_{E q, B 1}=d_{E q, B 2} \\
K_{E q, C B F}=K_{\text {frame }}+K_{E q, B 1}+K_{E q, B 2}\end{array}$ \\
\hline
\end{tabular}


The application of this model was used to extrapolate the $\mathrm{CBF}$ response from the experimental test results on the isolated U-connection. In the classical design and analysis of CBFs, the frame (beam, columns and beam-column connections) is modelled as an ideally hinged system therefore with no lateral stiffness. Thus, in a first stage, no contribution from the Frame component was considered. Figure 23 compares the lateral force versus lateral displacement response of extrapolated and experimental results on CBFs for two configurations. In order to be short, only two couple of connection-frame configurations are used. However, the model is valid for any configuration as long as the geometry of the U-plate is the same in both the connection and frame tests. Thus, from the experimental results on U-connections specimens 2 and 7, the response of the CBFs specimens 2 and 1 were respectively extrapolated. The results show that the experimental tests on the CBFs present a stiffer response than the model assuming no contribution of the Frame component. It is therefore clear (and expected) that the frame provides a contribution. Though the connections used between beam and columns are of a standard type, normally assumed as hinges, these do not represent perfect hinges and partially constrain the relative rotations between beam and columns. Thus a non-zero lateral stiffness is realistic to be assumed from the Frame component. In order to evaluate this contribution, one test was performed on a frame without any brace. The measured Frame lateral stiffness was $K=1.91 \mathrm{kN} / \mathrm{mm}$. Using this value, the extrapolation of the CBF response using the described model can be updated. Figure 24 presents the comparison of the experimental tests with the updated spring mechanical model. The comparison becomes then excellent.

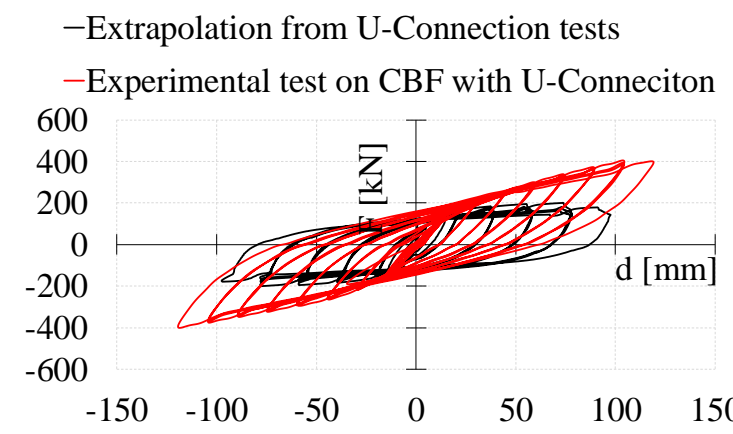

a) Test 2 vs CBF 2

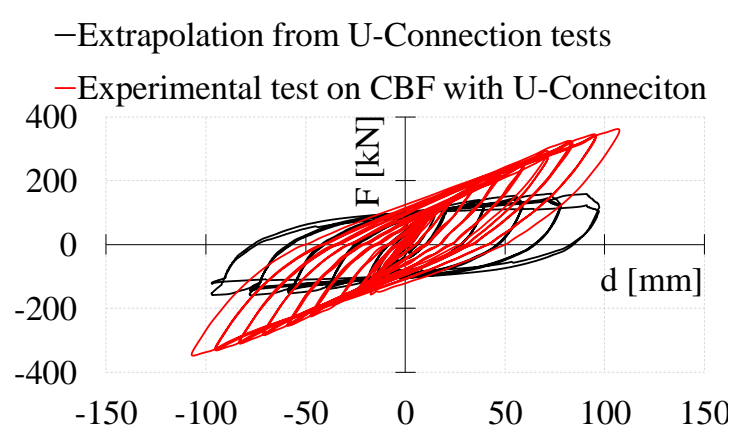

b) Test 7 vs CBF 1

Figure 23: U-connection response vs $\mathrm{CBF}$ response assuming ideally hinged frame
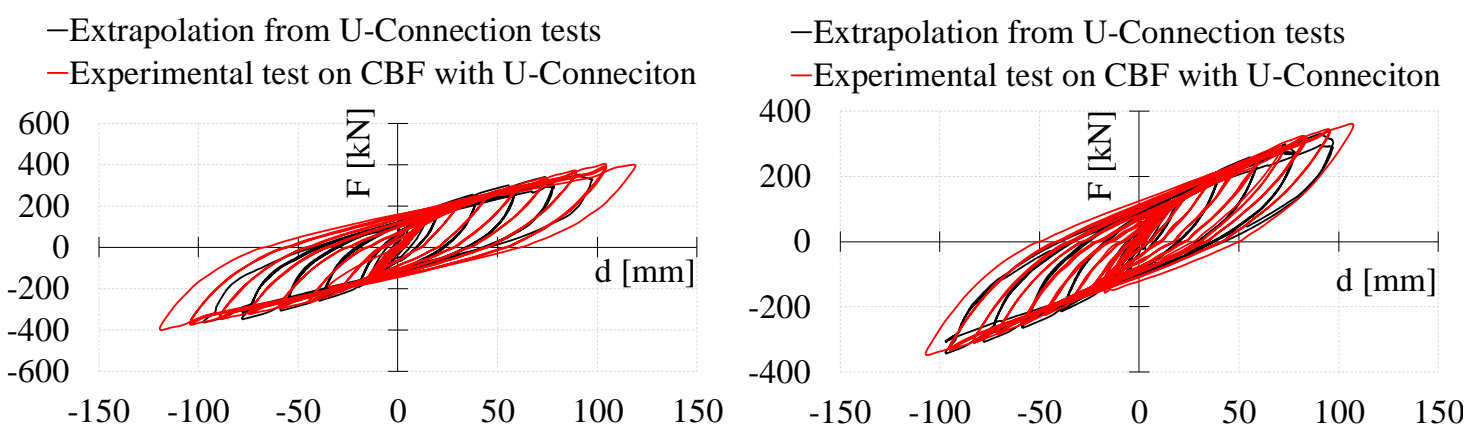

a) Test 2 vs CBF 2
b) Test 7 vs CBF 1

Figure 24: U-connection response vs CBF response including frame effect

In order to quantify the contribution of the U-connection to the global energy dissipation, the absorbed energy was compared for the tested frames with respect to a set of four Uconnections. Figure 25 depicts the cumulative absorbed energy at the end of the cycles for on the one hand the frame and on the other $4 \mathrm{U}$-connections as tested isolated. It shows clearly that the U-connections are responsible for almost the entire energy dissipation capacity of the tested frames, therefore confirming the efficiency of the U-connection as possible dissipative system in CBFs. On the other hand, the connection flexibility and deformability is also evident. This may result in high lateral deformations of the building structure when the system is applied on multi-storey buildings. Consequently, the second order effects may become significant and limit the suitability of the connection. In (Vayas I et al, 2017) the range of application of the U-connection on multi-storey buildings was studied and it was concluded that the connection is suitable for buildings up to 4 stories.

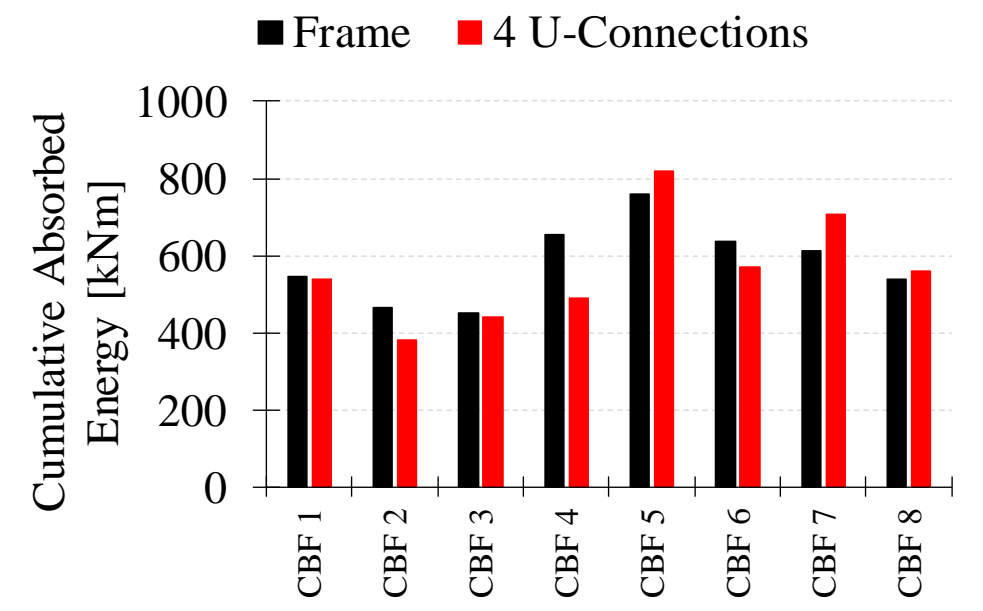

Figure 25: Cumulative Absorbed Energy Global Frame vs 4 U-connections

\section{Fatigue behavior of the U-connection}

\subsection{Fatigue design approach proposal}

The performance of the U-connection under cyclic loading demonstrated its sensitivity to low cycle fatigue, as shown in Figure 19. The comparison of the ultimate deformation capacity of the connection when subjected to either cyclic or monotonic loading resulted in a reduction of down to $65 \%$. The cyclic loading tests performed with constant amplitude allow identifying the cyclic behavior of the connection independently of the loading history. Based on these tests, fatigue design curves were derived and proposed for the fatigue design of U-connection following the design principles of EN 1993-1-9 (CEN, 2005a).

To model the fatigue behavior, the S-N line approach was used. The S-N line correlates the number of cycles $(N)$ with the nominal stress range $\Delta \sigma(S)$ and can be expressed as given in (1). In the Log-Log domain, expression (1) leads to (2). The latter represents a 
straight line with a slope equal to $-1 / \mathrm{m}$, known as the fatigue resistance line identifying the safe and unsafe regions.

$$
\begin{gathered}
N S^{m}=K \\
\log (N)=\log (K)-m \log (S)
\end{gathered}
$$

In equations (1) and (2), $N$ is the number of cycles to failure at constant stress range $S, m$ is a non-dimensional constant and $K$ is a dimensional parameter depending on the mechanical properties of the analysed detail.

Regarding the definition of the stress range, many proposals are available in the literature (Krawinkler H and Zohrei M , 1983; Ballio G and Castigloni C, 1995; Ballio G et al., 1997; Bernuzzi C et al., 1997). Here, the approach given in (Ballio G. and Castiglioni C, 1995) and (Bernuzzi C et al., 1997) was used. The authors adopted the Miner's rule, usually applied in high cyclic fatigue, and suggest that the same S-N curves are valid in high and low cycle fatigue if an equivalent stress range $\left(\Delta \sigma^{*}\right)$ is considered, associated with an ideal indefinitely elastic behavior of the material. Furthermore, global displacement parameters are used instead of local deformation parameters. This hypothesis validates formula (3). Subsequently, an effective stress range $\left(\Delta \sigma^{*}\right)$ associated with the real strain range, in an ideal component made of an indefinitely linear elastic material, can be determined as given in (4) and is taken as $\mathrm{S}$.

$$
\begin{aligned}
& \frac{\Delta \varepsilon}{\varepsilon_{y}}=\frac{\Delta v}{v_{y}} \\
& \Delta \sigma^{*}=\frac{\Delta v}{v_{y}} f_{y}
\end{aligned}
$$

Where:

- $\varepsilon$ - represents the strain,

- $\quad v$ - generalized displacement component (or rotation),

- $\Delta$ - range of variation in a cycle,

- the subscript $y$ identifies yielding of the material $\left(\varepsilon_{y}=f_{y} / E\right)$ as well as conventional yielding with reference to the generalized displacement component $\left(v_{y}\right)$ (or rotation $\theta_{y}$ ).

Finally, formula (1) can be re-written as follows:

$$
N\left(\frac{\Delta v}{v_{y}} f_{y}\right)^{m}=K
$$


For the prediction of low cycle fatigue endurance of steel structural details, it is appropriate to adopt a failure criterion based on parameters associated with the response of the component (e.g. stiffness, strength or dissipated energy). In the literature (Calado L and Azevedo J, 1989; Calado L and Castigloni C, 1996; Bernuzzi C et al., 2000), two failure criteria are available. Here the criterion proposed in (Calado L and Castigloni C, 1996) is used for the definition of the fatigue endurance $(N)$ as given in (6).

$$
W_{f} / W_{0} \leq \alpha
$$

Where:

- $W_{f}$ represents the ratio between the absorbed energy of the considered component at the last cycle before collapse and the energy that might be absorbed in the same cycle if it had an elastic-perfectly plastic behavior;

- $W_{0}$ is the same ratio but with reference to the first cycle in plastic range;

- $\alpha$ is a parameter determined by fitting results with experimental results.

The value of 0,5 recommended in (Calado L and Castigloni C, 1996) is used.

The slope of the line is defined by the parameter $m$ for which different values can be found in the literature (Krawinkler H and Zohrei M , 1983) (Ballio G et al., 1997). In (Ballio G et al., 1997) the authors propose a value of 3 while in (Krawinkler H and Zohrei M , 1983) a value of 2 is suggested. As proposed in (Ballio G. and Castiglioni C, 1995), a slope $m=3$ was used in the derivation of the best fitting S-N line to the experimental results.

Finally, according to the limit state design method, the parameter governing the design should be defined on the basis of statistical analysis referring to a given probability of failure $P_{f}$ (or of survival $1-P_{f}$ ). Consequently, the value $\log (k)$ was so defined that the line with a slope $(-1 / m)$ intersecting the $\mathrm{X}$-axis at $\log (k)$ is associated to a probability of $5 \%$ of test data falling below the line. The application of the described procedure to evaluate the low cycle fatigue behavior of the U-connection is discussed in the following section.

\subsection{Application and comparison with Eurocode 3 fatigue design approach}

According to the methodology described in $§ 6.1$, an $S-N$ line was first derived for each U-plate loading configuration, i.e. parallel and transverse. The division of the test results into two groups according to loading orientation is justified by a different behavior (see Figure 11). However, the resulting equations for best fit and, consequently suggested design curve, are very similar, as given in (7) and (8) respectively. Accordingly, the fatigue design of the U-connection should not make a distinction between both loading configurations. Figure 26 compares the experimental results with the proposed curves. In Figure 27, the test results on the U-plate are compared with the EN 1993-1-9 (CEN, 2005a) $S$ - $N$ lines for all detail categories. The results are in line with the proposed 
approach and clearly show that the U-plate is sensitive to low cycle fatigue. The higher is the stress amplitude, the lower is the number of cycles supported by the U-plate.

$$
\begin{aligned}
& \log N=12.00-3 \log S \\
& \log N=11.53-3 \log S
\end{aligned}
$$

Finally, Figure 28 presents different charts showing the influence of each test variable. Figure 28-a) compares the test specimens with different loading configuration and direction but with the same geometric properties. First it can be observed that the U-plate subjected to a transverse load (Tests 8 and 10) is stiffer, therefore the stress amplitude $(S)$ is higher. For the cases with the same stress amplitude, the results indicate that the Uplate loaded in parallel (Tests 5 and 6) present a better fatigue behavior, as this configuration shows a longer endurance. Figure 28-b) shows the effect of the U-plate angle. The tests compared are specimens $1\left(\alpha=45^{\circ}\right)$ vs $2\left(\alpha=50^{\circ}\right)$ for parallel loading and specimens $9\left(\alpha=39^{\circ}\right)$ vs $10\left(\alpha=45^{\circ}\right)$ for transverse loading. For the test specimens subjected to a transverse load, the fatigue behavior is better for an angle of $45^{\circ}$ than for $30^{\circ}$. For the specimens loaded in parallel, the results are very close to each other and it cannot be concluded which configuration presents a better fatigue performance. This is possibly related to a rather small in terms of angle between both tests $\left(45^{\circ}\right.$ vs $\left.50^{\circ}\right)$. With respect to the thickness of the U-plate, Figure 28-c) indicates that the lower is the thickness (Test 2 vs 3 and Test 9 vs 11), the better is the fatigue performance. Figure 28d) shows the results regarding the variation of the radius of the U-plate. The specimens compared are Test 1 vs 6 , Test 3 vs 4 and Test 2 vs 7 . The results indicate that the Uplates with a higher bent radius $(125 \mathrm{~mm})$ perform better under fatigue loading.

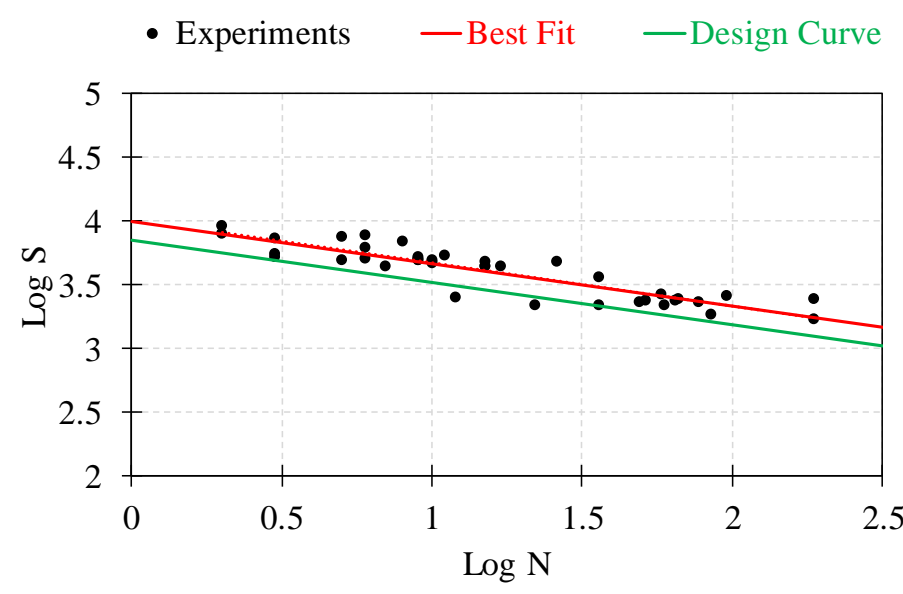

Figure 26: Best fit and low cycle fatigue design curves 


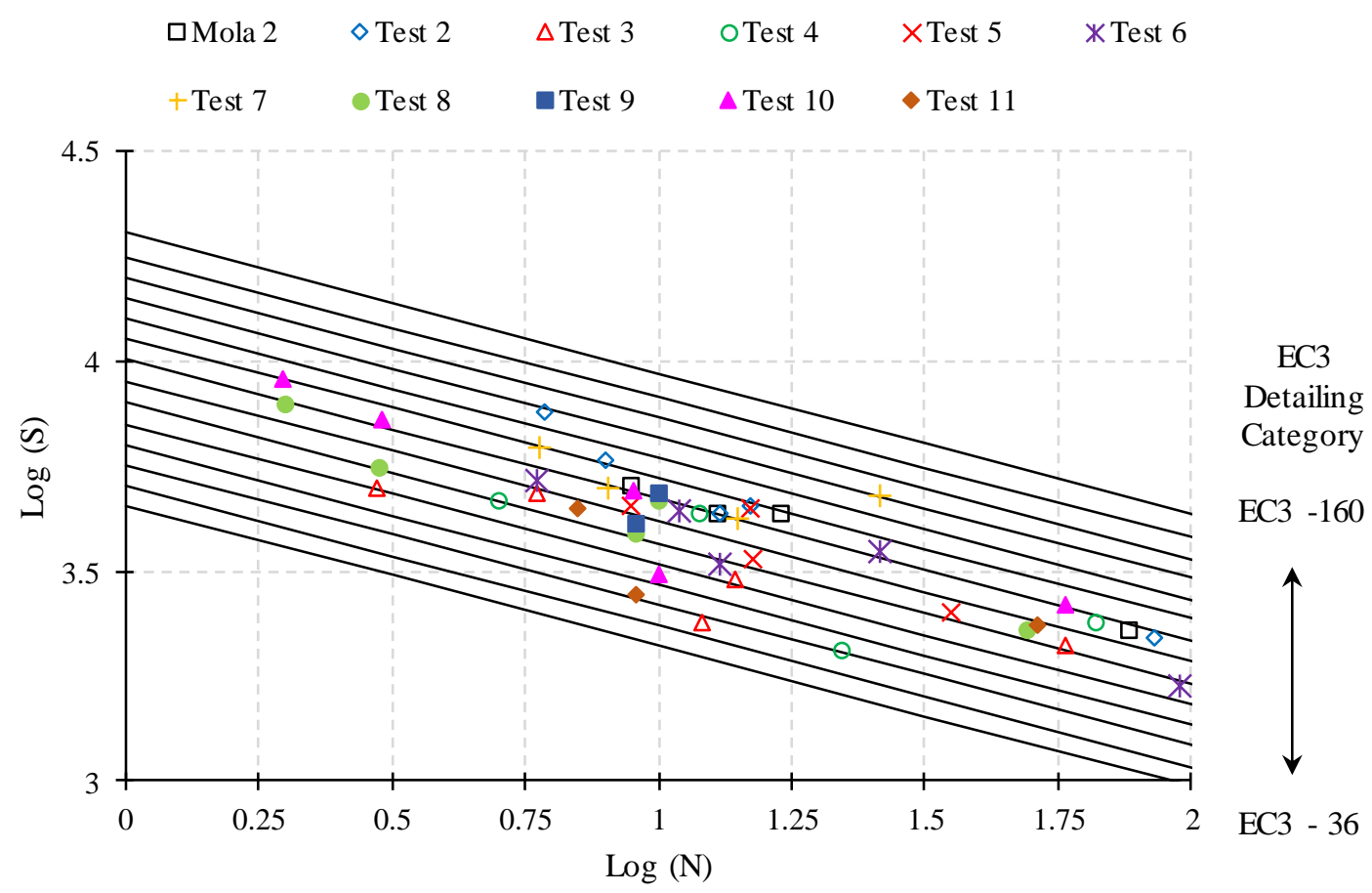

Figure 27: U-connection vs EC3 Fatigue line approach

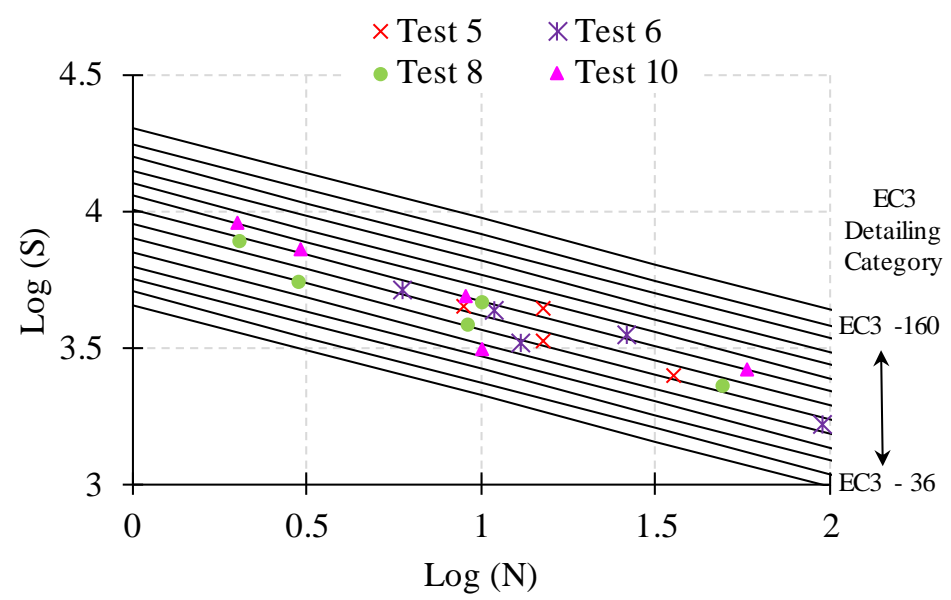

a) Loading direction

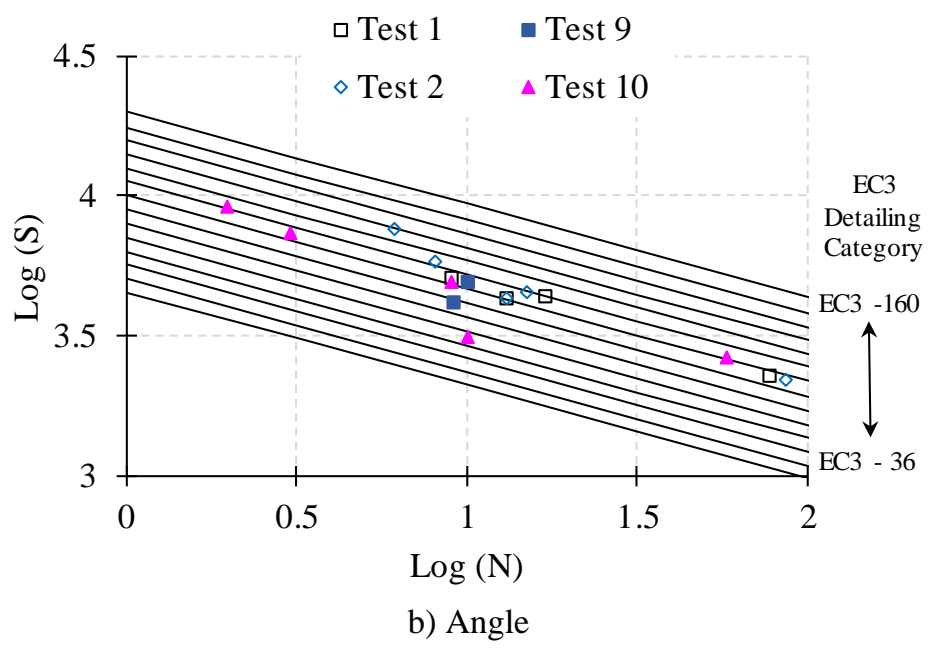




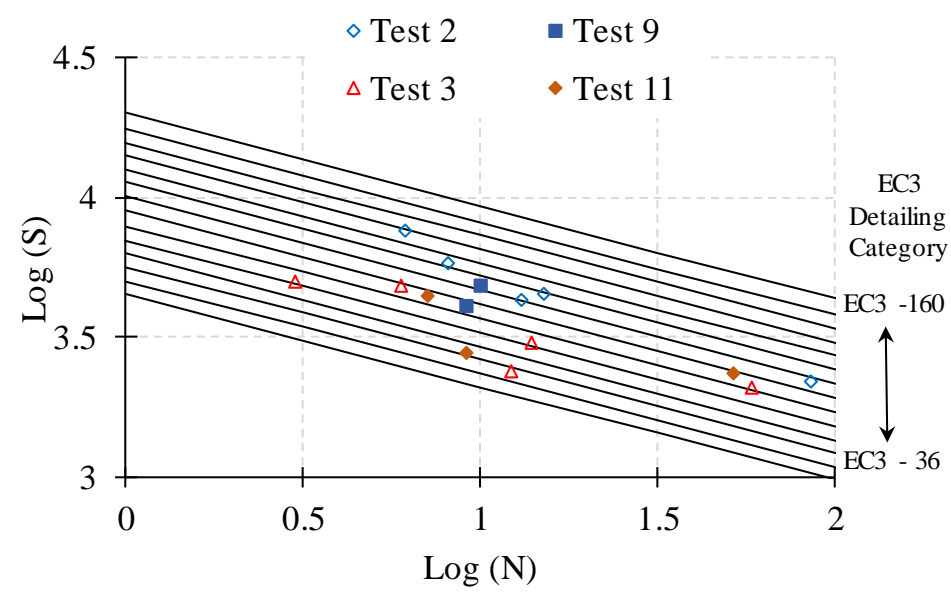

c) Thickness

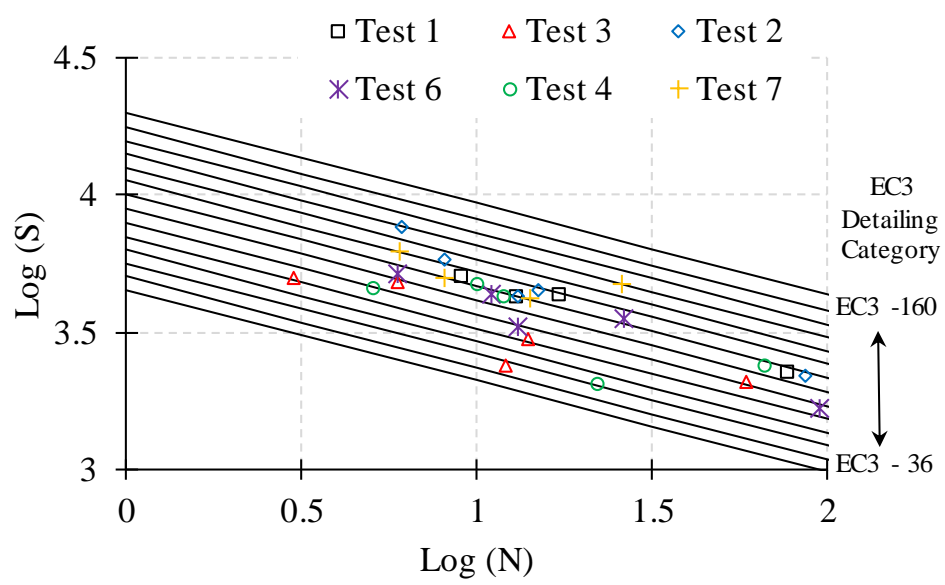

d) Bent radius

Figure 28: U-connection vs EC3 fatigue line approach comparing the different test variables

\section{Conclusions}

This paper presented an experimental programme on a dissipative connection (" $\mathrm{U}$ connection") foreseen for use in CBFs to connect braces to adjacent members. The connection consists in a U-shaped plate connected to braces and adjacent members by means of bolted connections. According to the configuration of the U-plate, the load transferred by the brace members is either parallel or transverse to the Plate "legs". The experimental programme contemplated tests on isolated connections and on one-storey real scale CBFs incorporating the U-connection. The varied parameters consisted in i) the load direction with respect to the U-plate (parallel or transverse, in tension or compression, ii) the load type, i.e. monotonic or cyclic, iii) the thickness of the U-plate, iv) the U-plate bent radius and v) the angle formed by the U-plate "legs", directly related to the global geometry of the frame.

From the analysis of the test results, the following conclusions are drawn:

- The response of the U-connection is asymmetric, the loading direction (respectively compression or tension in the brace) results in a different stiffness and strength of the connection. 
- The loading orientation with respect to the U-plate, i.e. parallel or transverse, has an impact on the connection response. When the load is applied transverse to the U-plate "legs" a stiffer and more resistant response is obtained. On the other hand, applying the load parallel to the U-plate "legs" results in a larger deformation capacity.

- The failure occurs in the net section of the bolted connection between the U-plate and the connected member. Before the failure is reached, significant inelastic flexural deformation develops however in the U-plate, leading to a high energy dissipation capacity.

- A U-plate with a lower bent radius, a higher thickness and subjected to a transverse load presents the best global performances.

- The cyclic tests demonstrate that the connection is potentially prone to low cycle fatigue. A significant reduction of the deformation capacity is observed when comparing monotonic to cyclic tests.

- Used in a concentrically braced frames designed according to the capacity design approach where the connection is considered as the dissipative element, the Uconnection demonstrates to be performant in dissipating the seismic energy input and therefore reducing actions effects. The connections are responsible for most of the dissipated energy.

- The non-symmetric response of the U-connection is not reflected at the global $\mathrm{CBF}$ scale if the U-connections are placed in a symmetric way within the frame.

- Although standard beam-column connection in CBF are usually idealized as perfect hinges, a non-negligible contribution of the frame structure to the stiffness is identified.

Then, given the evident impact of the cyclic loading on the U-connection deformation capacity, the experimental test results were further processed to characterize the fatigue behavior of the U-connection and to propose a practical design approach. The latter is based on the S-N line approach. Accordingly, best fit as well as design S-N lines are derived. An analysis of the effect of the various tested parameters on the S-N lines given in EN 1993-1-9 is presented. This comparison indicates that the best fatigue performance is obtained when the U-plate is subjected to a load parallel to the "legs, the angle steps away from $45^{\circ}$, the thickness is small and the bent radius is large.

Finally, although not being the main purpose of this paper and requiring additional investigations to tune some of the required parameters, suggestions can be made to handle the complex behavior of the connection, highly nonlinear and susceptible to low cycle fatigue, for the practical design of structural systems including such connections.

- Nonlinear behavior of the connection: For a predesign, a simplified modelling is suggested. An elasto-plastic behavior can be considered where the elastic stiffness if based on a secant stiffness rather than on the initial tangent stiffness, referring to a similar approach for other types of steel structures with non-linear connections (like for instance pallet racks). The most appropriate ratio between initial and secant stiffness should however be evaluated in further studies. In a 
second stage, a more accurate design can be accomplish by resorting to a nonlinear dynamic analysis considering the actual nonlinear response of the $\mathrm{U}$ connection.

- Fatigue behavior of the connection: For a predesign, a simplified and conservative approach can be implemented. The fatigue design stress can be extrapolated from an equivalent linear elastic analysis for seismic design, as prescribed in the EN 1998-1. Further, the number of cycles may be estimated based on the duration of the strong motion according to the magnitude of the target design earthquake, e.g. as proposed in (Novikova E I and Trifunac M D, 1994) and the fundamental vibration period of the structure. A less conservative and more accurate design requires the execution of nonlinear dynamic analysis.

\section{Acknowledgements}

The research leading to these results has received funding from the European Union's Research Fund for Coal and Steel (RFCS) research programme under CEC agreement $\mathrm{N}^{\circ}$ 7210-PR-316 (The INERD project) and the grant agreement $\mathrm{N}^{\circ} 709434$ (INNOSEIS).

\section{References}

Baird A et al. (2014). Experimental and numerical study of U-Shape Flexural Plate (UFP) dissipators. 2014 NZSEE Conference, (pp. P2-P11). Aotea Centre, Auckland.

Ballio $G$ et al. (1997). Low cycle fatigue and fracture of structural steel members and connections. Fatigue and Fracture of Engineering Materials and Structures, 20 (8), 1129-1146.

Ballio G. and Castiglioni C. (1995). A Unified Approach for the Design of Steel Structures under Low and/or Hugh Cycle Fatigue. Journal of Constructional Steel Research, 34 (1), 75-101.

Barroilhet A. (2011). Massive 8.8 Earthquake Hits the Tallest Building in Chile. The Anti Seismic Structure of Titanium La Portada Building. CTBUH 2011 World Conference. Seoul, Korea.

Bernuzzi C et al. (1997). Ductility and Load Carrying Capacity Prediction of Steel Beamto-Column Connections under Cyclic Reversal Loading. Journal of Earthquake Engineering, vol. 1, n.(2), 401-432.

Bernuzzi C et al. (2000). Low cycle fatigue of structural steel components: a method for re-analysis of test data and a design approach based on ductility. Indian Society of Earthquake Technology (ISET), Special Issue on Experimental Techniques, 37 (4), 47-63.

Bertero V et al. (1994). Performance of steel building structures during the Northridge earthquake. Berkley: Earthquake Engineering Research Center, University of California at Berkeley.

Calado L and Azevedo J. (1989). A model for predicting failure of structural steel elements. Journal of Constructional Steel Research, Vol. 14, 41-62. 
Calado L and Castigloni C. (1996). Steel beam-to-column connections under low-cycle fatigue experimental and numerical research. Proc. of XI World Conference on Earthquake Engineering. Acapulco, Mexico.

CEN. (2005a). EN 1993-1-9: Eurocode 3-Design of steel structures - Part 1-9: Fatigue. Brussels, Belgium: European Committee for Standardization.

CEN. (2005b). EN 1998-1: Eurocode 8 - Design of structures for earthquake resistance - Part 1: General rules, seismic actions and rules for buildings. Brussels, Belgium.: European Committee for Standardization.

Dimakogianni D et al. (2015). Seismic behavior of frames with innovative energy dissipation systems (FUSEIS1-2). Engineering Structures, Vol. 90, 83-95.

ECCS. (1986). Recommended testing procedure for assessing the behaviour of structural steel elements under cyclic loads. Brussels, Belgium: ECCS - Technical Committee 1 - Structural Safety and Loadings - Technical Working Group 1.3 Seismic Design.

Fardis M et al. (2005). Designers' Guide to EN 1998-1 and 1998-5. Eurocode 8: Design Provisions for Earthquake Resistant Structures. London, UK: Thomas Telford Publishing.

Hwang S-H and Lignos D G. (2017). Effect of modelling assumptions on the earthquakeinduced losses and collapse risk of steel-frame buildings with special concentrically braced frames. ASCE, Journal of Structural Engineering, Vol 143 (9), 04017116-1:04017116-16.

INERD. (2004). Two innovations for Earthquake resistant design, The INERD project. Brussels: Research Programme of the Research Fund for Coal and Steel.

Jensen H et al. (2012). Discrete variable structural optimization of systems under stochastic earthquake excitation. Structural Seismic Design Optimization and Earthquake Engineering: Formulations and Applications. United States: Vagelis Plevris, Chara Ch. Mitropoulou and Nikos D. Lagaros, Engineering Science Reference.

Kelly J et al. (1972). Mechanisms of Energy Absorption in Special Plates for use in Earthquake Resistant Structures. Bulletin of the New Zealand Society for Earthquake Engineering, 5(3), 63-88.

Krawinkler H and Zohrei M . (1983). Cumulative damage in steel structures subjected to earthquake ground motion. Computers \& Structures, 531-541.

Landolfo R et al. (2017). Design of Steel Structures for Buildings in Seismic Areas, 1st Edition, ECCS Eurocode Design Manuals. ECCS Press/Ernst\&Sohn.

Morelli F et al. (2017). Seismic behaviour of an industrial steel structure retrofitted with self-centering hysteretic dampers. Journal of Constructional Steel Research, 157175.

Novikova E I and Trifunac M D. (1994). Duration of strong ground motion in terms of earthquake magnitude, epicentral distance, site conditions and site geometry. Earthquake Engineering and Structural Dynamics 23 (9), 1023-1043.

Nüyün_tek. (2018). Nüyün_tek, a SIRVE company. 
Palermo A et al. (2005). Seismic Design of Multi-Storey Buildings using Laminated Veneer Lumber (LVL). 2005 New Zealand Society of Earthquake Engineering Conference, (pp. 14-22). Wairaki, New Zealand.

Pampanin S et al. (2011). Expectation Meets Reality: Seismic Performance of PostTensioned Precast Concrete Southern Cross Endoscopy Building During the 22nd Feb 2011 Christchurch Earthquake. New Zealand Concrete Industry Conference. Rotorua.

Pessiki S. (2017). Sustainable seismic design. Procedia Engineering 171, 33 - 39.

Priestley M J N. (2000). Performance Based Seismic Design. 12 WCEE 2000 : 12th World Conference on Earthquake Engineering (pp. 2831 - 2852). Auckland, New Zeland: New Zealand Society for Earthquake Engineering.

Priestley M J N et a.l. (1999). Preliminary Results and Conclusions from the PRESSS Five-story Precast Concrete Test-Building. PCI Journal, Vol. 44(6), 42-67.

Schultz A et al. (1994a). Experimental study of joint connections in precast concrete walls. Proceedings of the Fith U. S. National Conference on Earthquake Engineering, Vol. II, (pp. 579-587).

Schultz A et al. (1994b). Seismic resistance of vertical joints in precast shear walls. Proceedings of the Fith U. S. National Conference on Earthquake Engineering, Vol. I,, (pp. E23-E27).

Tan K G and Christopoulos C. (2016). Developement of replaceable cast steel links for eccentrically braced frames. ASCE, Journal of Structural Engineering, Vol 142 (10), X:Y.

Vayas I et al. (2017). Innovative Anti-seismic Plates and systems. Ioannis Vayas (Ed.), Research Fund for Coal and Steel, INNOSEIS Project RFCS-02-2015, ECCS press. 